\title{
KPNB1-mediated nuclear translocation of PD-L1 promotes non-small cell lung cancer cell proliferation via the Gas6/MerTK signaling pathway
}

\author{
Wenwen $\mathrm{Du}^{1,2} \cdot$ Jianjie $\mathrm{Zhu}^{1,2,3} \cdot$ Yuanyuan Zeng ${ }^{1,2,3} \cdot$ Ting Liu $^{1,2} \cdot$ Yang Zhang $^{1,2} \cdot$ Tingting Cai ${ }^{1,2} \cdot$ Yulong Fu $^{1,2}$. \\ Weijie Zhang ${ }^{1,2} \cdot$ Ruochen Zhang ${ }^{1,2} \cdot$ Zeyi Liu $^{1,2,3} \cdot$ Jian-an Huang ${ }^{1,2,3}$
}

Received: 12 April 2020 / Revised: 13 October 2020 / Accepted: 15 October 2020 / Published online: 2 November 2020

(c) The Author(s) 2020. This article is published with open access

\begin{abstract}
In addition to the role of programmed cell death ligand 1 (PD-L1) in facilitating tumour cells escape from immune surveillance, it is considered as a crucial effector in transducing intrinsic signals to promote tumour development. Our previous study has pointed out that PD-L1 promotes non-small cell lung cancer (NSCLC) cell proliferation, but the mechanism remains elusive. Here we first demonstrated that PD-L1 expression levels were positively correlated with pMerTK levels in patient samples and NSCLC cell lines. In addition, PD-L1 knockdown led to the reduced phosphorylation level of MerTK in vitro. We next showed that PD-L1 regulated NSCLC cell proliferation via Gas6/MerTK signaling pathway in vitro and in vivo. To investigate the underlying mechanism, we unexpectedly found that PD-L1 translocated into the nucleus of cancer cells which was facilitated through the binding of Karyopherin $\beta 1$ (KPNB1). Nuclear PD-L1 (nPDL1), coupled with transcription factor Sp1, regulated the synthesis of Gas6 mRNA and promoted Gas6 secretion to activate MerTK signaling pathway. Taken together, our results shed light on the novel role of nPD-L1 in NSCLC cell proliferation and reveal a new molecular mechanism underlying nPD-L1-mediated Gas6/MerTK signaling activation. All above findings provide the possible combinational implications for PD-L1 targeted immunotherapy in the clinic.
\end{abstract}

These authors contributed equally: Wenwen Du, Jianjie Zhu,

Yuanyuan Zeng

Edited by S. Kaufmann

Supplementary information The online version of this article (https:// doi.org/10.1038/s41418-020-00651-5) contains supplementary material, which is available to authorized users.

$\triangle$ Zeyi Liu

liuzeyisuda@163.com

$\triangle$ Jian-an Huang

huang_jian_an@163.com

1 Department of Pulmonary and Critical Care Medicine, The First Affiliated Hospital of Soochow University, 215006 Suzhou, China

2 Suzhou Key Laboratory for Respiratory Diseases, 215006 Suzhou, China

3 Institute of Respiratory Diseases, Soochow University, 215006 Suzhou, China

\section{Introduction}

As an important member of the B7 protein family, programmed cell death ligand 1 (PD-L1) is well known for its role in facilitating tumour cells escape from immune surveillance through its binding with receptor programmed cell death-1 (PD-1), which expressed on immune cells [1, 2]. In recent years, despite the considerable clinical improvement that has been achieved with immunotherapy targeting PDL1/PD-1 checkpoints, a durable response to these therapies was only observed in a minority of patients [3, 4]. In addition to the immunosuppressive properties, accumulated studies have indicated that PD-L1, as a membrane receptor, could transduce intrinsic signals to promote tumour development, differentiation and metabolism $[5,6]$.

Earlier in 2008, convincing evidence identified that PDL1 expressed in P815 and Renca cancer cells made them resistant to $\mathrm{T}$ cell cytotoxicity even if $\mathrm{T}$ cells expressed a signal-null PD-1 [7]. A growing body of evidence has demonstrated that PD-L1 exerts initial oncogenic effects in mediating tumour proliferation, metastasis, autophagy, glucose metabolism and drug resistance [8-10]. It has been 
reported that in BRAFV600E human colon cancer cells, PDL1 expression was increased, thereby inducing BIM and BIK to enhance chemotherapy-induced apoptosis [11]. In melanoma and ovarian cancer cells, PD-L1 transduced cellintrinsic signals to regulate immune-independent tumour growth, mTOR signaling and autophagy [12]. Our previous studies have found that PD-L1 can induce NSCLC cells resistant to EGFR-TKIs via TGF- $\beta /$ Smad signaling pathway [9]. Moreover, we reported that knockdown of PD-L1 inhibited tumour cell proliferation and induced apoptosis in HCC827 and PC9 cell lines [13]. However, how PD-L1 transduced the intrinsic signals and the involved signalosome remained to be illuminated.

The distribution of PD-L1 differs among tumour specimens. PD-L1 is mainly located in the cell membrane and cytoplasm, while it also exists in the nucleus to a less extent. Positive nuclear PD-L1 (nPD-L1) expression is identified in surgically resected renal cell carcinomas, lung cancer and hepatocellular carcinomas tissues [14]. In human oesophageal cancer, nPD-L1 expression is significantly correlated with tumour invasion depth [15]. In colorectal cancer and prostate cancer, nPD-L1 expression in circulating tumour cells has been identified to be significantly correlated with progressionfree survival or overall survival, suggesting that nPD-L1 could be a potential prognostic biomarker in cancer patients [16]. In addition, it has been reported that doxorubicin treatment increased nPD-L1 expression via the phosphorylation of AKT in breast cancer [17]. Similarly, PD-L1 translocation played a major role in enhancing tumour cell radioresistance in head and neck squamous cell carcinoma [18]. However, it is still unclear how PD-L1 enters the nucleus and the function of nPD-L1 in NSCLC is rarely explored.

Karyopherin $\beta 1$, also known as KPNB1, is an important nuclear receptor which involved in shuttling proteins from the cytoplasm to nucleus. It is reported that KPNB1mediated NUAK1 nuclear translocation was inhibited by oxidative stress [19]. In addition, the presence of KPNB1 is related with tumour progression. In breast cancer, suppression of KPNB1 inhibited cancer cell proliferation by abrogating nuclear transport of Her2 [20]. KPNB1 knockdown can inhibit chronic myeloid leukaemia cell proliferation and induce cell apoptosis via regulation of E2F1 [21]. In colorectal cancer, KPNB1 deficiency suppressed tumour cell growth and metastasis by reducing MET expression [22]. Consistently, our previous study proved that increased KPNB1 expression promoted NSCLC cells proliferation and induced chemoresistance via the PI3K /AKT pathway [23]. These data implicated that KPNB1 could be a potential target for future therapies in cancer; however whether KPNB1 involved in the PD-L1 translocation need to be elucidated.

Tyro3, Axl and MerTK comprise the TAM family of receptor tyrosine kinases (RTKs) [24]. Activation of MerTK signaling by its ligands Growth Arrest-Specific 6 (Gas6) and Protein S1 (PROS1) promoted cell proliferation and metastasis and suppressed the immune response in tumour microenvironment [25-27]. The downstream signaling includes the PI3K/AKT, MAPK/ERK, NF-KB, and JAK/ STAT pathways $[26,28]$. In glioblastoma, higher expression of MerTK is found to be positively related to tumour TNM stage and malignance, leading to poorer survival for patients [29]. Recent studies demonstrated that MerTK is over-expressed in lymphoma and melanoma and the inhibition of MerTK leads to suppression of tumour growth via AKT and Erk signaling [30, 31]. Moreover, MerTK activation promotes drug resistance to $E G F R$ tyrosine kinase inhibitors in NSCLC [32].

In our current study, we have shown that tumour PD-L1 activated intra-tumour cell Gas6/MerTK signals which promoted NSCLC cell proliferation in vitro and in vivo. For the first time, we proved that KPNB1, as an intracellular PD-L1binding partner, facilitated the nuclear translocation of PD-L1 in NSCLC. Furthermore, nPD-L1, coupled with transcription factor Sp1, transcriptionally regulated Gas6 mRNA synthesis and promoted the secretion of Gas6 to activate MerTK signaling which enhanced NSCLC proliferation. The identification of nPD-L1 redefined its role in cancer development through Gas6/MerTK axis, which provided an alternative view of developing potential therapies for patients who are refractory to PD-L1 targeted immunotherapy.

\section{Materials and methods}

\section{Patient samples}

A total of 24 paired NSCLC tissue specimens were collected in the Department of Pulmonary and Critical Care Medicine, First Affiliated Hospital of Soochow University. All participants were provided with written informed consent at the time of recruitment. All samples were kept at $-80{ }^{\circ} \mathrm{C}$ for storage. All cases had clinically and pathologically confirmed diagnoses of NSCLC based on the Revised International System for Staging Lung Cancer. The present study was approved by the Ethics Committee of the First Affiliated Hospital of Soochow University. In addition, 52 paired NSCLC tissues and adjacent lung tissues were used for the construction of tissue microarray (Outdo Biotech, Shanghai, China). The detailed information for patients is listed in Tables S1 and S2.

\section{Haematoxylin-eosin staining (H\&E) and immunohistochemical (IHC) assay}

The detailed procedures for $\mathrm{H} \& \mathrm{E}$ and $\mathrm{IHC}$ analysis were conducted as described in our previous study [33] and 
also mentioned in our supplementary materials. The antibodies used in our current study were as follows: PD-L1 (2B11D11, ProteinTech Group Inc., Chicago, IL, USA), PD-L1 (28-8, Abcam, Cambridge, UK), PD-L1 (E1L3N, Cell Signaling Technology, Danvers, MA), p-MerTK (ab192649, Abcam, Cambridge, UK) and Ki67 antibody (8D5, Cell Signaling Technology, Danvers, MA).

\section{Cell culture, transient transfection and establishment of stable cell lines}

HCC827 and H1299 cells were purchased from the Cell Bank of the Chinese Academy of Sciences (Shanghai, China). All cell lines were authenticated by STR profiling. Cells were maintained in RPMI-1640 medium, except HEK 293T cells were cultured in DMEM (Sigma, South Logan, UT, USA). All media had $10 \%$ foetal bovine serum (Gibco, South America, SA, USA) and 1\% antibiotics (Beyotime, Shanghai, China). For siRNA or plasmid transient transfection, cells were seeded in 6well plates until they reached $40-60 \%$ confluence and then transfected with the expression vectors or specific siRNAs targeting Sp1 or MerTK using JetPRIME reagent (Life Technologies). The construction methods of stable PD-L1 knockdown and overexpression cell lines were reported in our previous study [9]. For the generation of KPNB1-overexpressing cells, a 2619-bp fragment of the KPNB1 coding sequence was synthesised (Genewiz, Suzhou, China) and subcloned into the PLVX-IRES-Neo vector using the endonucleases SwaI and NotI. The methods were exactly the same as those performed in the construction of stable PD-L1overexpressed cells. To generate stable KPNB1 knockout cell lines, gRNAs targeting KPNB1 cloned into plentiCRISPR v2 were synthesised by Genewiz Company. The target sequences were listed in Table S3. Then the KPNB1 gRNA constructs along with the packaging plasmids were co-transfected into HEK 293T cells using Lipofectamine 3000 (Invitrogen) for $48 \mathrm{~h}$. Then we collected the supernatant to infect cancer cells at least three times, followed by puromycin selection. All detailed methods were shown in supplementary materials.

\section{RNA extraction and quantitative real-time PCR analysis}

These procedures were performed as previously mentioned [34]. All PCR primer sequences used for PD-L1, Gas6, MerTK, PROS1, Sp1, CDK1, CDK4 and $\beta$-actin mRNA detection; the primers for construction of the Gas6 or Sp1 promoter; and other primers, including Gas6 ChIP primers and primers for KPNB1 stable cell lines or Sp1 overexpression, are listed in Table S3.

\section{Cellular fractionation, western blot and co- immunoprecipitation assay}

Cellular fractions were isolated using the nuclear protein extraction kit (R0050, Solarbio Life Science). Generally, cells were first lysed with cytoplasmic protein extraction buffer, followed by nuclear extraction buffer to obtain the nuclear fraction. In some conditions, after serum starvation for $24 \mathrm{~h}$, PD-L1-overexpressed cells were treated with $3 \mu \mathrm{M}$ UNC2025 (APExBIO, B8016) or $10 \mu \mathrm{M}$ LDC1267 (APExBIO, B4893) for $48 \mathrm{~h}$. The PD-L1 knockdown cells were stimulated with $500 \mathrm{ng} / \mathrm{ml}$ Gas6 (R\&D Systems, 885GSB) for $24 \mathrm{~h}$. The antibodies used in the analysis were anti-PD-L1 (2B11D11), anti-Tubulin (1E4C11), anti-Lamin B1 (ProteinTech Group Inc., Chicago, IL, USA); anti-Bcl-2 (sc-7382), anti-MerTK (sc-365499) (Santa Cruz, CA, USA); anti-KPNB1 (ab2811), anti-p-MerTK (Y749 + Y753 + Y754) (ab14921, Abcam, Cambridge, UK); anticyclinD1 (92G2), anti-AKT(11E7), anti-pAKT (Ser473) (D9E), anti-ERK (137F5), anti-pERK (Thr202/Tyr202) (D13.14.4E), anti-Sp1 (D4C3) (Cell Signaling Technology, Danvers, MA, USA); anti-Gas6 (A8545) (Abclonal, Boston, USA); anti-Flag (Sigma, St, Louis, MO, USA); anti- $\beta$ actin and anti-mouse or -rabbit secondary antibodies (Cell Signaling Technology). For immunoprecipitation assays, protein lysates were incubated with anti-PD-L1, antiKPNB1, anti-Flag or the normal IgG antibody at $4{ }^{\circ} \mathrm{C}$ overnight with rotation. At day 2, the mixture was further incubated with protein $\mathrm{A} / \mathrm{G}$ beads or M2 anti-Flag resin at room temperature for $2-3 \mathrm{~h}$. After washing with lysis buffer three times, the beads were boiled and then followed by western blot assay.

\section{CCK-8 and clonogenic assay}

Cells $\left(3 \times 10^{3}\right)$ were seeded in 96-well plates. At $24 \mathrm{~h}, 48 \mathrm{~h}$ and $72 \mathrm{~h}$, Cell Counting Kit-8 solution (Boster, Wuhan, China) was added to each well, and the OD value was determined by measuring the absorbance at $450 \mathrm{~nm}$ and $630 \mathrm{~nm}$. For clonogenic assays, cells were cultured for 7-10 days until the formation of foci. Then, the cells were fixed with methanol, stained with Giemsa and counted.

\section{5-Ethynyl-2'-deoxyuridine (EdU) assay}

The EdU assay kit was purchased from Ribobio Company (C10310-1, Guangzhou, China). Generally, $3 \times 10^{3}$ cells were added to 96 -well plates and cultured for $24-48 \mathrm{~h}$ to reach 50-60\% confluence. The cells were exposed to fresh medium containing $50 \mu \mathrm{M}$ EdU and cultured in the incubator for an additional $2 \mathrm{~h}$. The cells were fixed with $4 \%$ paraformaldehyde and then permeabilized with $0.5 \%$ Triton $\mathrm{X}-100$. They were stained with $100 \mu \mathrm{l}$ Apollo reaction 
cocktail for $30 \mathrm{~min}$, followed by $100 \mu \mathrm{l}$ Hoechst 33342 $(5 \mu \mathrm{g} / \mathrm{mL})$ incubation for $5 \mathrm{~min}$. An Olympus IX-73 inverted microscope (Tokyo, Japan) was used to capture the images.

\section{Cell cycle and apoptosis analysis}

PD-L1-overexpressed cells and the control cells were treated with UNC2025 $(3 \mu \mathrm{M})$ for $48 \mathrm{~h}$. The cell cycle and apoptosis analysis kit was purchased from Beyotime Biotechnology (Shanghai, China). For the cell cycle analysis, cells were washed with cold PBS and then fixed in $70 \%$ ethanol at $4{ }^{\circ} \mathrm{C}$ overnight. Then the cells were stained with propidium iodide (PI)/RNase mixture in the dark at $37^{\circ} \mathrm{C}$ for $30 \mathrm{~min}$. For the apoptosis assay, both supernatant and cells were harvested, washed, and suspended in binding buffer containing Annexin V/FITC or APC and PI (Beyotime, Shanghai, China). Fluorescence-activated cell sorting (FACS) of stained cells was done in a FACSCalibur system (Beckman Coulter, Brea, CA, USA).

\section{Immunofluorescence staining}

Cells were seeded in 12-well plates that were pre-inserted with glass slides. Twenty-four hours later, when the cells had reached $40-50 \%$ confluence, they were washed with PBS. Cells were then fixed with $4 \%$ paraformaldehyde for $30 \mathrm{~min}$ followed by permeabilization with $0.5 \%$ Triton X-100 solution for an additional $20 \mathrm{~min}$. Next, $5 \%$ bovine serum albumin was added to function as the blocking buffer. The primary antibodies used in our experiment were anti-PD-L1 (1:200, Abcam) and anti-KPNB1 (1:200, Abcam). The corresponding secondary antibodies tagged with $\mathrm{Cy} 3$ and FITC were used (1:500, Beyotime Biotechnology).

\section{Luciferase reporter assay}

H1299-PLVX- and PD-L1-overexpressed cells were transiently transfected with the indicated pGL3-luciferase reporter $(500 \mathrm{ng}$ ) containing the $\mathrm{Sp} 1$ promoter or different truncated regions of the Gas6 promoter, along with the Renilla pRL-TK plasmid (40 ng) set as the inner control. After $24-48 \mathrm{~h}$, the cell lysates were subjected to further luciferase activity examination with a Dual-Luciferase Reporter Assay kit (Promega).

\section{ELISA}

H1299- and HCC827-PD-L1-overexpressed cells were exposed to medium containing $1 \%$ FBS for $12 \mathrm{~h}$ and then treated with the following inhibitors separately: the autophagy inhibitor 3-methyladenine (1 mM, Selleck, S2767), the protein transport inhibitor brefeldin A $(10 \mathrm{ng} / \mathrm{ml}$, APExBIO,
B1400), and the exosome secretion inhibitor 5-(N,N-dimethyl)-amiloride DMA (50 nM, APExBIO, C3505). The supernatant was collected from the cultured medium $24 \mathrm{~h}$ later. Gas6 level was measured with a human Gas6 ELISA kit (SEA204Hu, USCN Life Sciences, Wuhan, China).

\section{Mass spectrometry assay}

The affinity-purified samples were analysed with the Orbitrap Elite hybrid mass spectrometer (Thermo Fisher) coupled with a Dionex LC according to the instructions. Briefly, the cell lysates from HEK 293T cells transfected with human pcDNA3.1-Flag-PD-L1 plasmid and the control were excised and processed for MS analysis. Samples were loaded onto an enrichment column and separated in an analytical column over 120 min with an LC gradient from 6 to $44 \%$ solvent B $(80 \%$ acetonitrile, $0.1 \%$ formic acid). Mass spectra were acquired in the positive-ion mode with an automated data-dependent tandem MS analysis in the CID mode for the 15 most intense ions from each precursor MS scan. Each selected precursor ion was analysed twice in $60 \mathrm{~s}$. The resolution for the precursor ion was set to 120,000 , and the MS/MS spectra were collected for the selected precursor ion within a $2 \mathrm{Da}$ mass isolation window.

\section{Chromatin immunoprecipitation (ChIP) assay}

A ChIP-IT kit (Active Motif, Carlsbad, CA, USA) was used to perform the ChIP assay. Briefly, cells were fixed with formaldehyde and then lysed. To precipitate the DNA fragment, $2 \mu \mathrm{g}$ anti-Sp1 or anti-PD-L1 antibody or normal IgG were used. The DNA-protein complexes were pulled down with magnetic beads and then de-crosslinked. The extracted DNA samples were then amplified with specific Gas6 promoter primers.

\section{In vivo tumour xenograft animal model}

UNC2025 was administered to 4-6-week-old female BALB/c athymic nude mice. The model was established using HCC827-PD-L1 or control cells suspended in PBS and $3 \times$ $10^{6}$ cells were inoculated subcutaneously into the flanks of 20 nude mice. When the tumour weight reached nearly $100 \mathrm{~mm}^{3}$, 10 mice injected with HCC827-PD-L1 cells were randomly divided into two groups with or without UNC2025. And the same was done for the rest 10 mice injected with control cells. UNC2025 was administrated via oral gavage at a dose of $75 \mathrm{mg} / \mathrm{kg}$ once a day for 24 days. Tumour weight and tumour volume were monitored every other day. All the animal experiments were carried out in complete compliance with the Guide for the Care and Use of the Experimental Animal Centre of Soochow University. 


\section{Statistical analysis}

All experiments were independently performed in triplicate as a minimum. All statistical analyses were done using GraphPad Prism 7.0 (GraphPad, San Diego, CA, USA) and SPSS 17.0 software (SPSS, Chicago, IL, USA). All data were presented as mean \pm SD. Significant differences between two groups were assessed by a nonpaired Student's $t$ test. Significant differences between three or more groups were analysed using one-way or two-way ANOVA analysis followed by Bonferroni post hoc test. All statistical tests were two-tailed. $P<0.05$ was set as statistically significant.

\section{Results}

\section{The role of PD-L1 in mediating NSCLC cell proliferation in vitro}

To validate the role of PD-L1 in cell proliferation, we first steadily knocked down or overexpressed PD-L1 in H1299 and HCC827 cell lines. The altered PD-L1 mRNA and protein expression levels were confirmed in these two cell lines (Fig. 1a, b). To assess the effect of PD-L1 in cancer cell proliferation, CCK-8 assay was utilised. PD-L1 knockdown inhibited cell growth (Fig. 1c). In contrast, cell growth was significantly enhanced in PD-L1overexpressed cells (Fig. 1d). We next confirmed this finding via clonogenic assay (Fig. 1e, f). The EdU assay was performed to further verify the above results. Consistently, the percentage of EdU-positive cells was decreased after PD-L1 knockdown (Fig. 1g), while an increased percentage of EdU-positive cells was observed in the PD-L1-overexpressed group (Fig. 1h). Collectively, these data suggested that the presence of PD-L1 promotes NSCLC cell proliferation.

\section{PD-L1 is positively correlated with p-MerTK expression level in NSCLC tissues}

To investigate the underlying mechanism, human RTK phosphorylation array was performed to detect the specific tyrosine kinase targets of PD-L1. The data showed that the phosphorylation of MerTK was significantly downregulated after PD-L1 knockdown (Fig. S1a, c). Other changed kinases were shown in Table S4 and Fig. S1b. We first verified the association between PD-L1 and p-MerTK expression in 24 paired NSCLC tissues and adjacent lung tissues. Among these samples, 17 pairs showed consistently up-regulated PD-L1 and p-MerTK expression in NSCLC tissues. Five pairs demonstrated reduced PD-L1 and p-MerTK expression in NSCLC tissues. Other two cases were presented with non-correlated expression in PD-L1 and p-MerTK (Fig. 2a, b). Further correlation assay showed a positive association between PD-L1 and pMerTK protein levels (T/N) (Fig. 2c). Moreover, the IHC assay proved that NSCLC tissues with increased PD-L1 expression showed higher p-MerTK expression (Fig. 2d). Individual positive or negative controls were shown in Fig. S2. These findings indicated a positive correlation between PD-L1 and p-MerTK expression levels in NSCLC tissue.

\section{Tumour intrinsic PD-L1 regulates NSCLC cell proliferation through Gas6/MerTK signaling}

We also verified the association between PD-L1 and pMerTK expression in NSCLC cell lines. The expression of p-MerTK was down-regulated in PD-L1 knockdown cell lines. Meanwhile, p-AKT, p-Erk, Cyclin D1, and Bcl-2 were markedly reduced, while the total AKT and Erk levels were not changed (Fig. 3a). In contrary, PD-L1 overexpression up-regulated p-MerTK, p-AKT, p-Erk, Cyclin D1 and Bcl-2 expression levels in both H1299 and HCC827 cell lines (Fig. 3b). To further validate the role of MerTK and its associated downstream signaling pathway in PD-L1-mediated cell proliferation, PD-L1overexpressed and control cells were treated with the MerTK selective inhibitor UNC2025. PD-L1overexpression rescued UNC2025-induced decreased percentage of cells in the G0/G1 and $S$ phases and increased percentage of cells in the G2/M phase when compared to control group (Fig. S3a). Further, we found that UNC2025 treatment resulted in an increase in cell apoptosis, while PD-L1 overexpression reduced the UNC2025-mediated apoptosis as evidenced by a lower cell apoptosis rate (Fig. S3b). In addition, PD-L1 overexpression rescued the decreased expression levels of $\mathrm{p}$ MerTK, p-AKT, p-Erk, Cyclin D1 and Bcl-2 after UNC2025 treatment, which was in accordance with the flow cytometry results (Fig. 3c). Moreover, we observed similar results in cells which were treated with two individual MerTK specific siRNAs (Fig. 3e). LDC1267 is a widely used inhibitor for TAM signaling pathway. We found that PD-L1 overexpression rescued the reduced expression levels of p-MerTK, p-AKT, p-Erk, Cyclin D1 and Bcl-2 after LDC1267 treatment when compared to control cells (Fig. 3d). To further confirm these findings, we activated MerTK with rh-Gas6 in PD-L1 knockdown cell lines. PD-L1 knockdown blocked Gas6-induced MerTK signaling activation compared to control cells (Fig. 3f). These results strongly suggested that PD-L1 controls tumour cell proliferation at least partially by regulating the cell cycle and apoptosis via MerTK signaling pathway. 
A

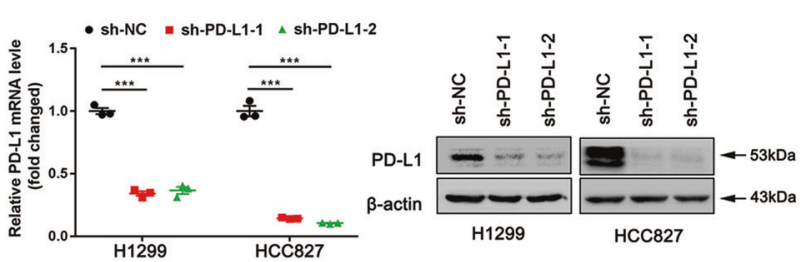

C

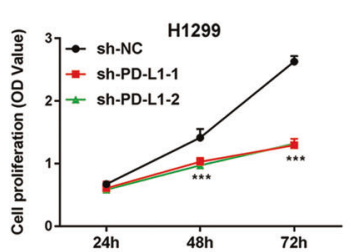

E

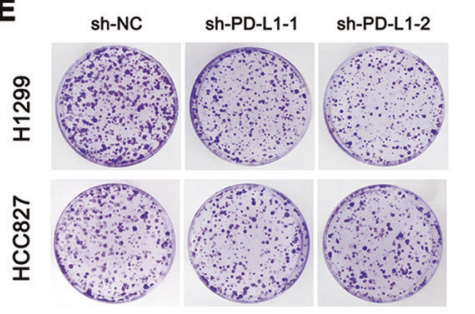

\section{G}
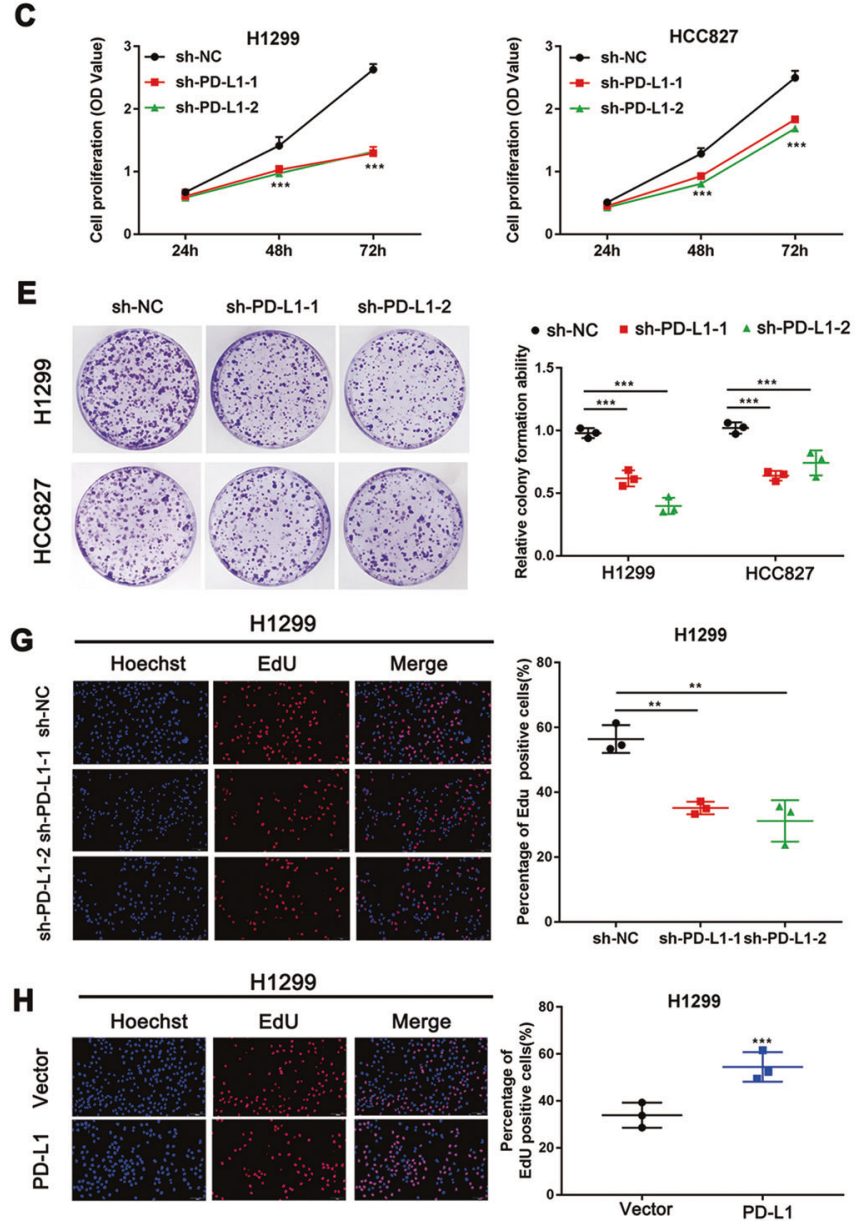

Fig. 1 The role of PD-L1 in mediating NSCLC cell proliferation in vitro. a, b The mRNA and protein levels in H1299 and HCC827 cell lines after PD-L1 knockdown or overexpression. c, d CCK-8 analysis of $\mathrm{H} 1299$ and HCC827 cell viability under PD-L1 silencing or overexpression. e, $\mathbf{f}$ Representative images of the clonogenic assay of $\mathrm{H} 1299$ and HCC827 cell proliferation under PD-L1 silencing or

\section{Inhibition of MerTK signaling blocks PD-L1- overexpression induced tumour growth in murine xenograft model}

Then we extended our study to murine subcutaneous xenograft models by administering UNC2025 in vivo. HCC827 cells with stable PD-L1 overexpression or control cells were inoculated into BALB/C athymic mice. Tumour growth was significantly inhibited in mice treated with $75 \mathrm{mg} / \mathrm{kg}$ UNC2025 in both PD-L1-overexpressed mice and control mice. However, the tumour sizes were much larger
B

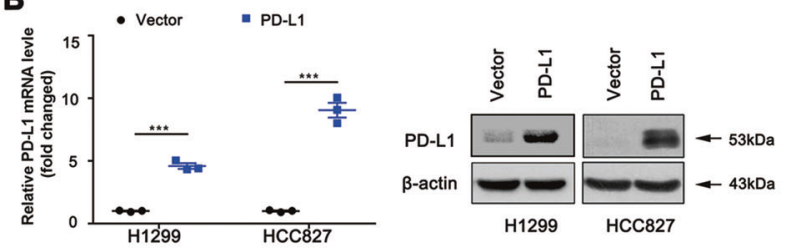

D
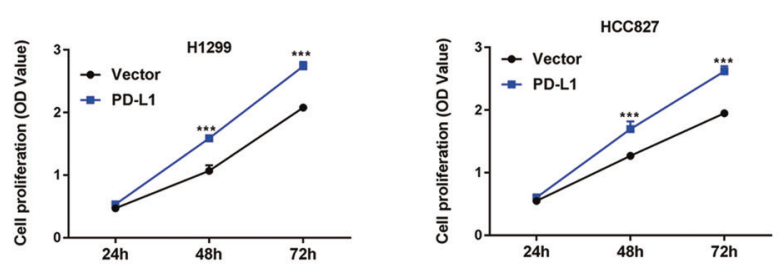

$\mathbf{F}$
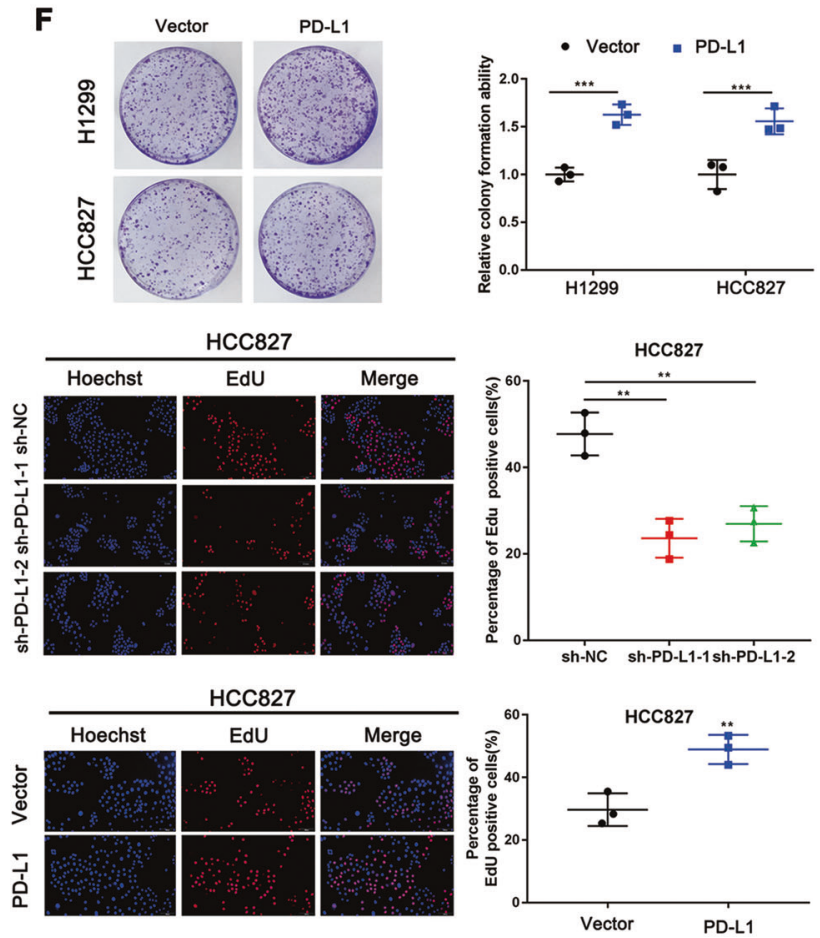

overexpression. g, h Representative images of the EdU assay in H1299 and HCC827 cells with PD-L1 knockdown or overexpression (Scale bar: $2 \mathrm{~mm}$ ). Data were presented as the mean \pm SD. Data were analysed using non-paired Student's $t$ test, one-way or two-way ANOVA analysis followed by Bonferroni's post hoc test. $* * P<0.01$, $* * * P<$ 0.001 vs. control or as indicated.

in the PD-L1-overexpressed mice compared to control mice when both groups were administrated with equal amount of UNC2025 (Fig. 4a, b). The changes in tumour weight were in line with the tumour volume (Fig. 4c). The tissues resected from the xenograft tumour were then lysed for western blot assay. PD-L1 overexpression rescued the decreased activation of MerTK and downstream AKT and Erk signaling after UNC2025 treatment (Fig. 4d). We then confirmed the above findings through analysing the pMerTK and Ki67 expression levels in these groups (Fig. 4e). Collectively, all above data indicated that tumour 
A
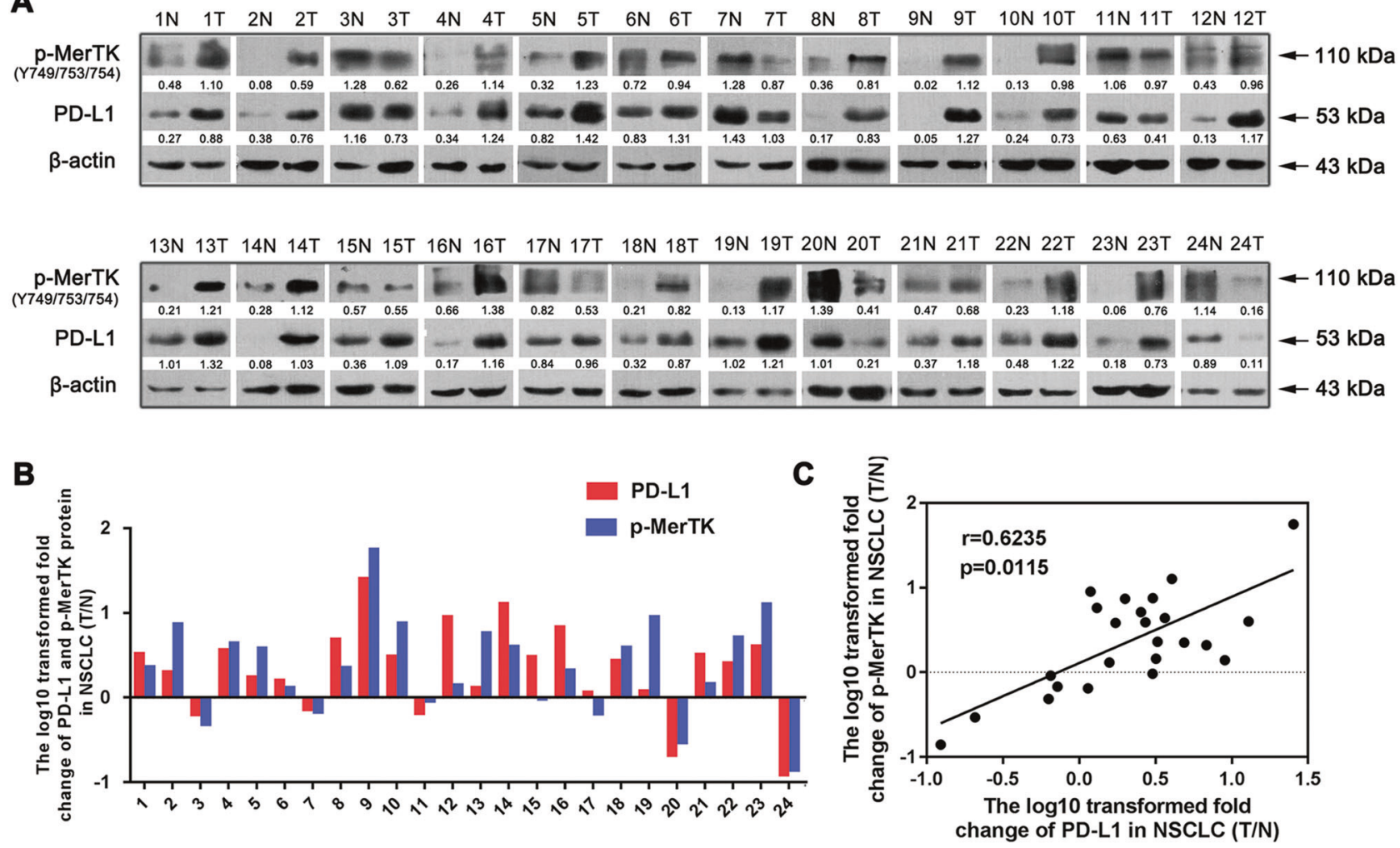

D

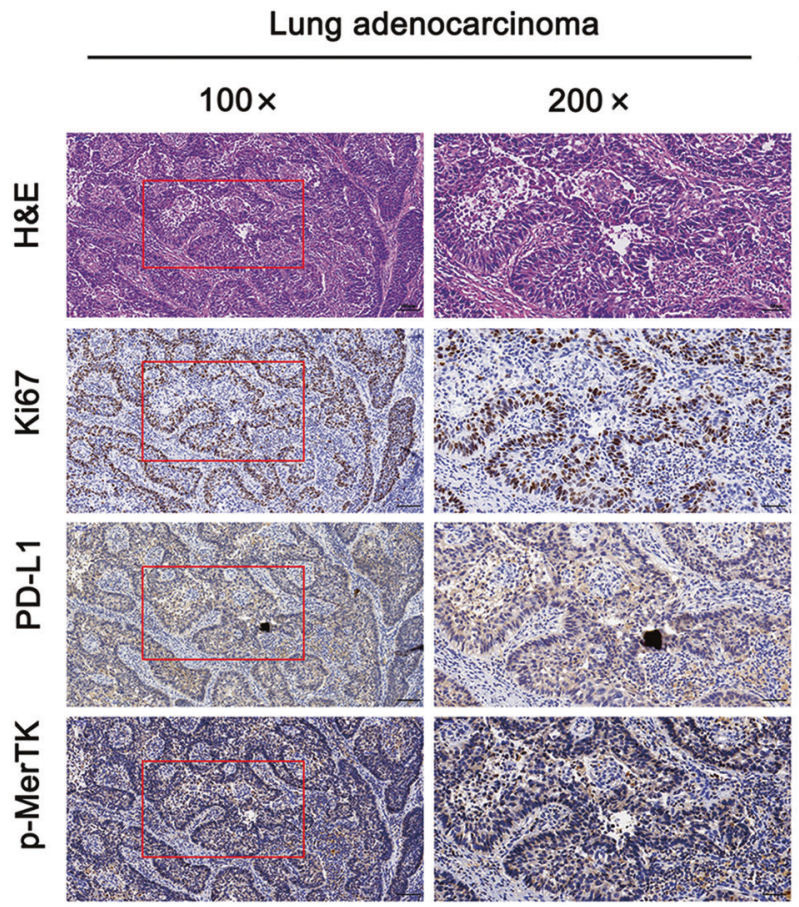

Fig. 2 PD-L1 is positively correlated with p-MerTK protein levels in human NSCLC tissues. a Western blot assay of PD-L1 and p-MerTK protein levels in 24 paired NSCLC tissues and the corresponding adjacent tissues. b Relative quantification of PD-L1 and p-MerTK protein levels in 24 paired NSCLC tissues. The $Y$ axis refers
Lung squamous cell carcinoma

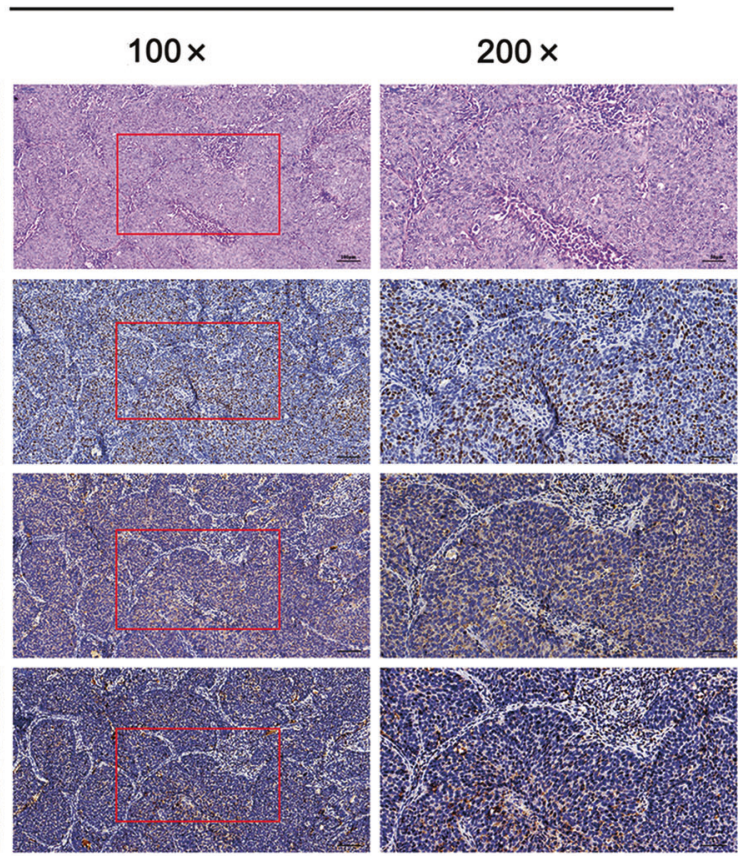

to the $\log 10$-transformed fold change of the $\mathrm{T} / \mathrm{N}$ expression ratio. The $X$ axis represents sample number. c The correlation between PD-L1 and p-MerTK protein levels in 24 paired NSCLC tissues. d IHC assay of PD-L1 and p-MerTK protein expression in NSCLC tissues (Scale bar: $100 \mu \mathrm{m}, 50 \mu \mathrm{m})$. 

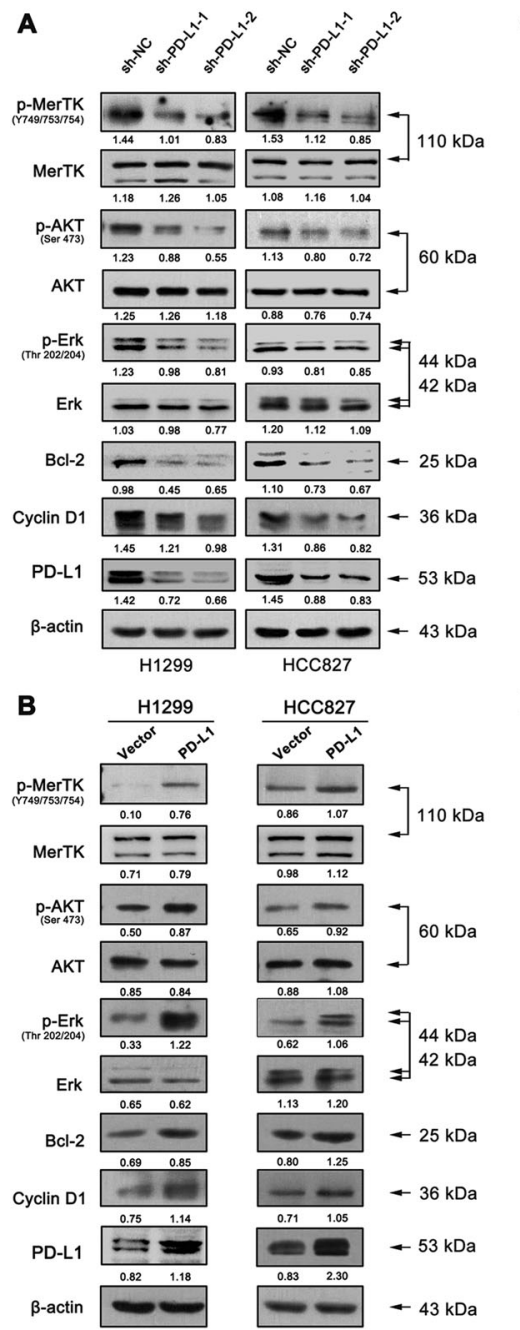

Fig. 3 Tumour intrinsic PD-L1 regulates NSCLC cell proliferation through Gas6/MerTK signaling. a, b Western blot assay of PD-L1, p-MerTK, MerTK, p-AKT, AKT, p-Erk, Erk, Bcl-2 and Cyclin D1 expression in stable PD-L1 knockdown or overexpressed cells when compared to control cells. c, d PD-L1-overexpressed and control cells were treated with $3 \mu \mathrm{M}$ UNC2025 or $10 \mu \mathrm{M} \mathrm{LDC} 1267$ for $48 \mathrm{~h}$, then the cell lysates were proceed with western blot assay to test PD-L1,

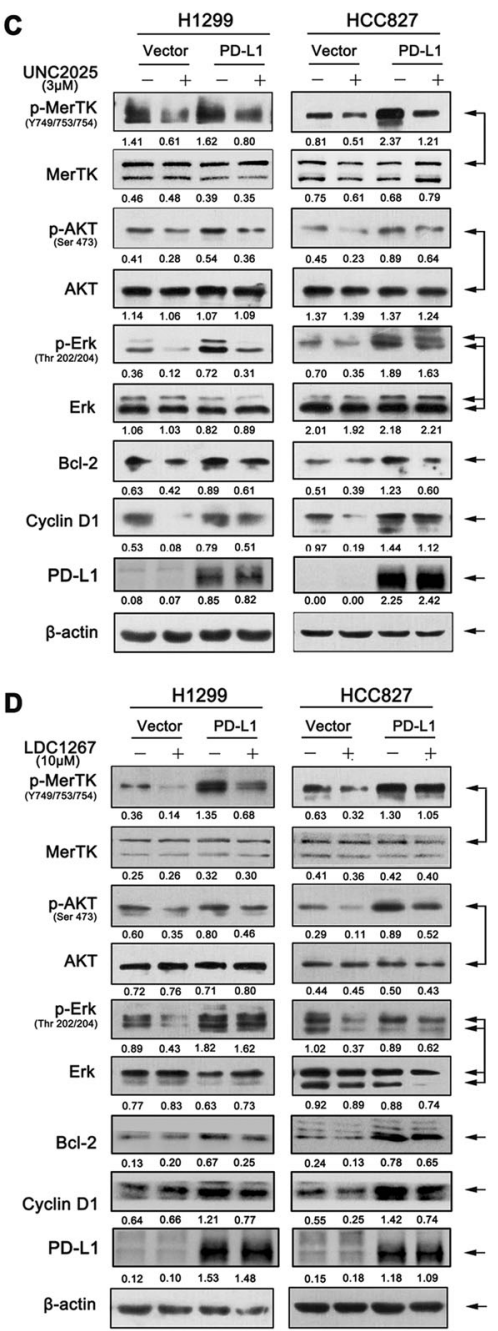

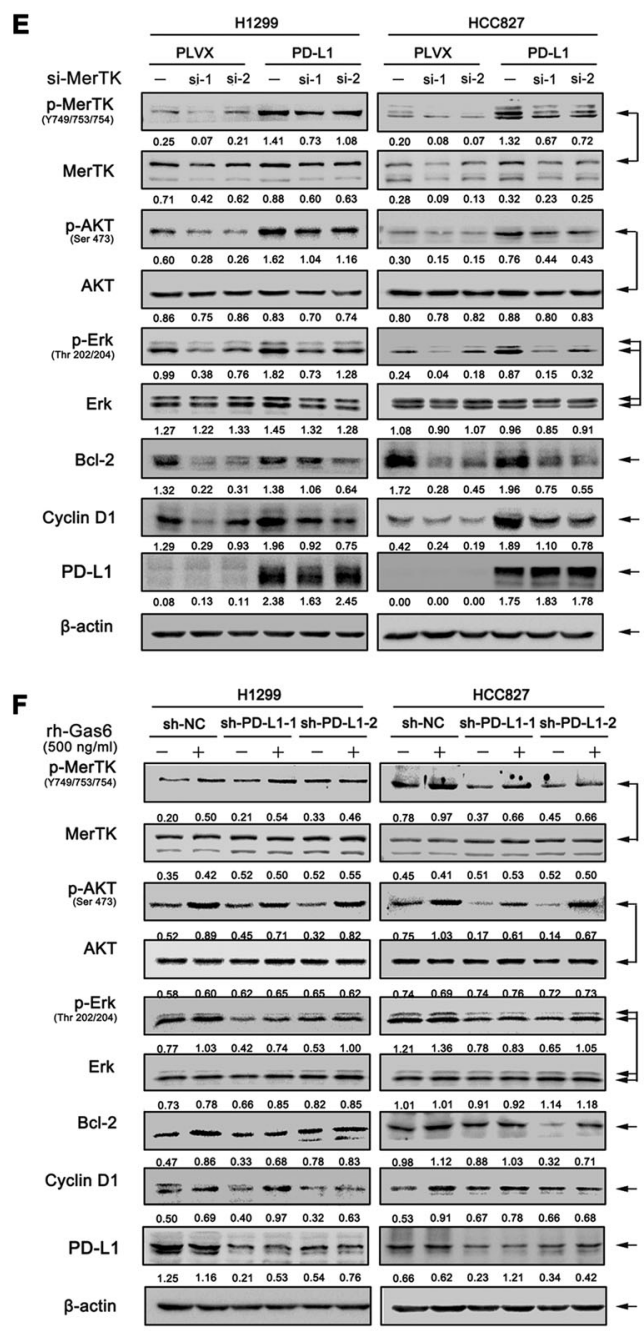

p-MerTK, MerTK, p-AKT, AKT, p-Erk, Erk, Bcl-2 and Cyclin D1 protein levels. e Western blot assay of the above proteins in PD-L1 overexpressing cells after knocked down with two separate siMerTKs. f Western blot assay of p-MerTK, MerTK, p-AKT, AKT, p-Erk, Erk, Bcl-2 and Cyclin D1 protein levels in PD-L1 stable knockdown cells after stimulated with $500 \mathrm{ng} / \mathrm{ml}$ rh-Gas6 for $24 \mathrm{~h}$. intrinsic PD-L1 promotes cell proliferation via Gas6/MerTK signaling in vitro and in vivo.

\section{Tumour intrinsic PD-L1 regulates Gas6 transcription and secretion}

Next, we sought to determine the mechanism that how MerTK phosphorylation was regulated by PD-L1. It is well known that MerTK signaling is activated via binding with its ligands Gas6 and PROS1. Therefore, we tested whether PD-L1 could regulate Gas6 or PROS1 expression. QRT-PCR data showed that Gas6 mRNA expression was down-regulated in PD-L1 knockdown cell lines, while PD-L1 overexpression up-regulated Gas6 expression at the transcriptional level (Fig. 5a). In term of PROS1, no significant changes were found after PD-L1 knockdown or overexpression (Fig. 5b). Interestingly, ELISA data found that manipulation of PD-L1 expression regulated Gas6 secretion (Fig. 5c). It has been reported that Gas6 can bind to phosphatidylserine-positive vesicles, such as exosomes, and act as exosome-derived ligands to activate TAMs [35]. We verified this mechanism by applying the autophagy inhibitor 3-MA, the Golgi apparatus transport inhibitor brefeldin $\mathrm{A}$ and the exosome biogenesis inhibitor DMA. Only DMA treatment showed a blocking effect on the secretion of Gas6, suggesting that Gas6 can be secreted via the exosome (Fig. S4). All above data suggested that PD-L1 promoted 


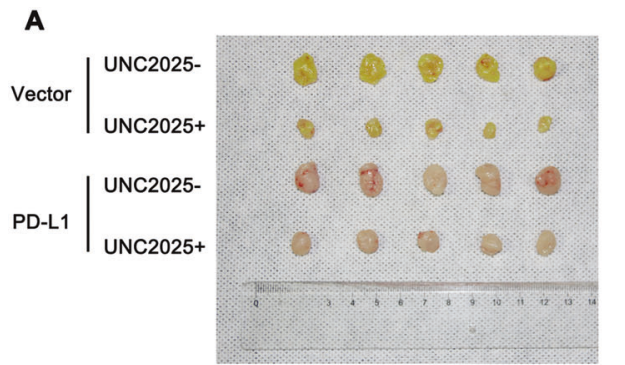

D

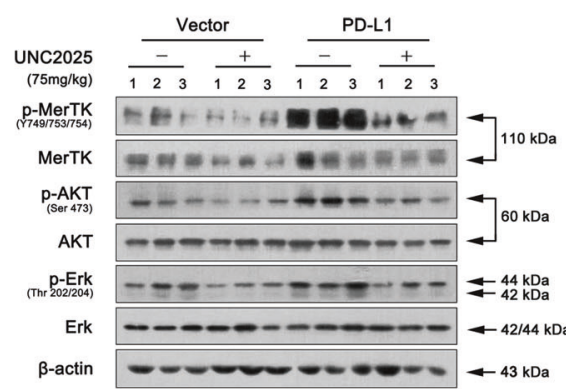

B

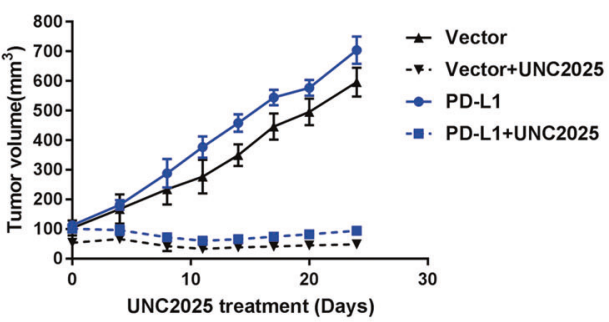

E

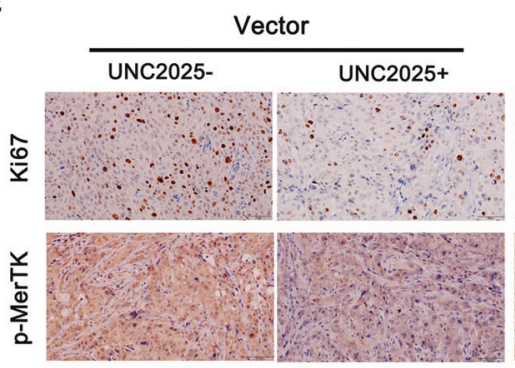

C
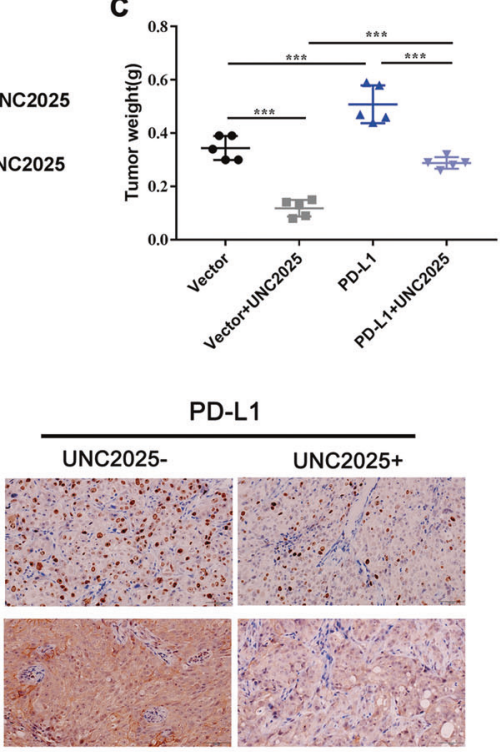

Fig. 4 Inhibition of MerTK signaling blocks PD-L1-overexpression induced tumour growth in murine model. a Representative images of tumours in these four groups. See the Methods for details. b, c Tumour volume and weight in the PD-Lloverexpression and control groups with or without UNC2025 treatment. d Western blot assay of
p-MerTK, MerTK, p-AKT, AKT, p-Erk, Erk, Bcl-2 and Cyclin D1 protein levels in these four groups. e IHC assay of KI-67 and p-MerTK expression (Scale bar: $100 \mu \mathrm{m})$. Data were presented as the mean \pm SD. Data were analysed using two-way ANOVA analysis followed by Bonferroni's post hoc test. $* * * P<0.001$ vs. control or as indicated.
Gas6 transcription and secretion, which may contribute to MerTK activation.

\section{KPNB1 is critical for PD-L1 nuclear translocation}

PD-L1 is widely recognised to be located in the cytoplasm and cell membrane. A few studies have also shown that the nPD-L1 was observed in surgically resected lung adenocarcinomas and lung squamous cell carcinomas tissues [14]. Based on our finding that manipulation of PD-L1 regulated the expression and secretion of Gas6, and this raised the possibility that PD-L1 might enter the nucleus to promote Gas6 transcription. First, we used three different clones of anti-PD-L1 antibodies to detect nPD-L1 expression. In addition to 2B11D11 clone used in current study, another universally recommended anti-PD-L1 antibody (clone 28-8) was utilised to stain the same tissue sections (Fig. 6a). It was reported that nPD-L1 could be detected by using antiPD-L1 antibody (clone E1L3N) [14]. So next we sought to detect nPD-L1 in our 52 paired NSCLC tissue microarray using clone E1L3N antibody. Among these 52 paired tissues, 17 cases showed positive nPD-L1 staining. And we randomly selected representative 6 cases to show nPD-L1 expression (Fig. S5a). Taken together, we found positive nPD-L1 expression in NSCLC tissues using three different anti-PD-L1 antibodies, which is in line with the published results [14]. Confocal microscopy analysis showed nPD-L1 location in NSCLC parental cells
(Fig. 6b). We further verified the presence of nPD-L1 by separating the cytoplasmic (non-NE) and nuclear (NE) fractions from PD-L1-overexpressed and control cells. We also noticed that nPD-L1 expression level was increased in PD-L1-overexpressed cells (Fig. 6c), which was further supported by confocal microscopy data (Fig. 6d). Similar results were obtained from HEK293T and Hela cells, which were transfected with Flag-PD-L1 plasmid (Fig. 6e).

Recently, it was reported that external factors, such as hypoxia, irradiation, and chemotherapeutic agents had effects on the distribution of PD-L1 within the cells $[16,17]$. To test whether PD-L1 can enter the nucleus of NSCLC cells under the treatment of paclitaxel and cisplatin, cytoplasmic and nuclear fraction of treated cells was isolated and then subjected to western blot analysis. Interestingly, paclitaxel significantly promoted the nuclear translocation of PD-L1 compared to that of cisplatin (Fig. S5b, c).

To gain insight into the underlying mechanism of PD-L1 nuclear import process, we performed mass spectrometry analysis in human HEK 293T cells to determine the potential interacting partners of PD-L1. We found that KPNB1 was one of the most strongly associated proteins with PD-L1 (Figs. 7a). To further test this finding, coimmunoprecipitation assay was performed and endogenous interaction of PD-L1-KPNB1 complex was confirmed in H1299 and HCC827 cells (Fig. 7b and S6a). We further coexpressed myc-tagged KPNB1 and Flag-tagged PD-L1 in 
A

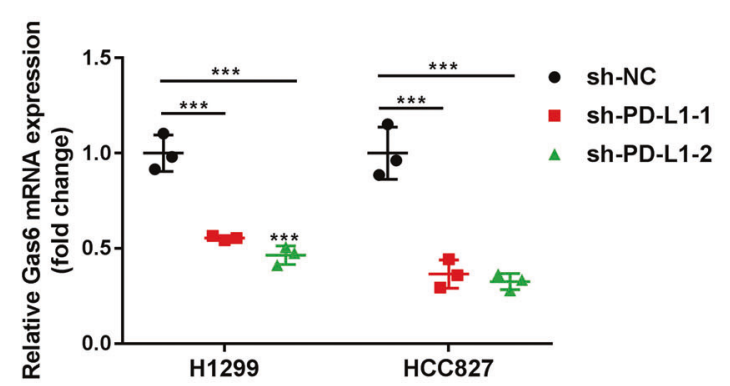

B

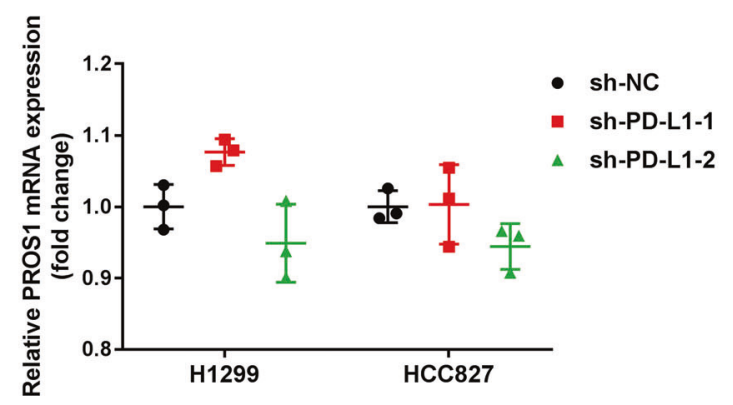

C

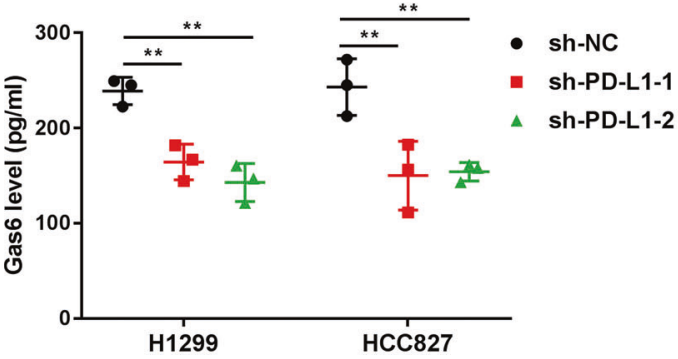

Fig. 5 Tumour intrinsic PD-L1 promotes Gas6 transcription and secretion. a, b qRT-PCR analysis of Gas6 and PROS1 mRNA expression levels in stable PD-L1 knockdown and overexpression cell lines. $\mathbf{c}$ The basal secretion level of Gas6 in PD-L1 stable cell lines and

HEK 293 T cells to verify the above finding. PD-L1 was detected in KPNB1 immunoprecipitates, indicating that PDL1 interacted with KPNB1 (Fig. 7c). As shown in Fig. 7d, KPNB1 was co-localised with membrane, cytoplasmic and nuclear PD-L1 via confocal microscopy analysis (Fig. 7d). Public database showed also that KPNB1 expression was positively correlated with PD-L1 in NSCLC tissues (Fig. 7e). To investigate whether the process of PD-L1 nuclear translocation was affected by KPNB1, two siRNAs were individually used to knockdown the expression of KPNB1, and PD-L1 nuclear translocation was inhibited (Figs. 7f and S6b), which was further supported by immunofluorescence assays (Fig. $7 \mathrm{~g}$ ). In addition, similar result was obtained in PD-L1-overexpressed cell lines in which KPNB1 was genetically modified using CRISPR/ Cas9 (Fig. 7h). In contrast, overexpression of KPNB1 promoted PD-L1 nuclear accumulation (Fig. 7i). Taken together, the above data suggested that KPNB1 is responsible for PD-L1 nuclear transport.
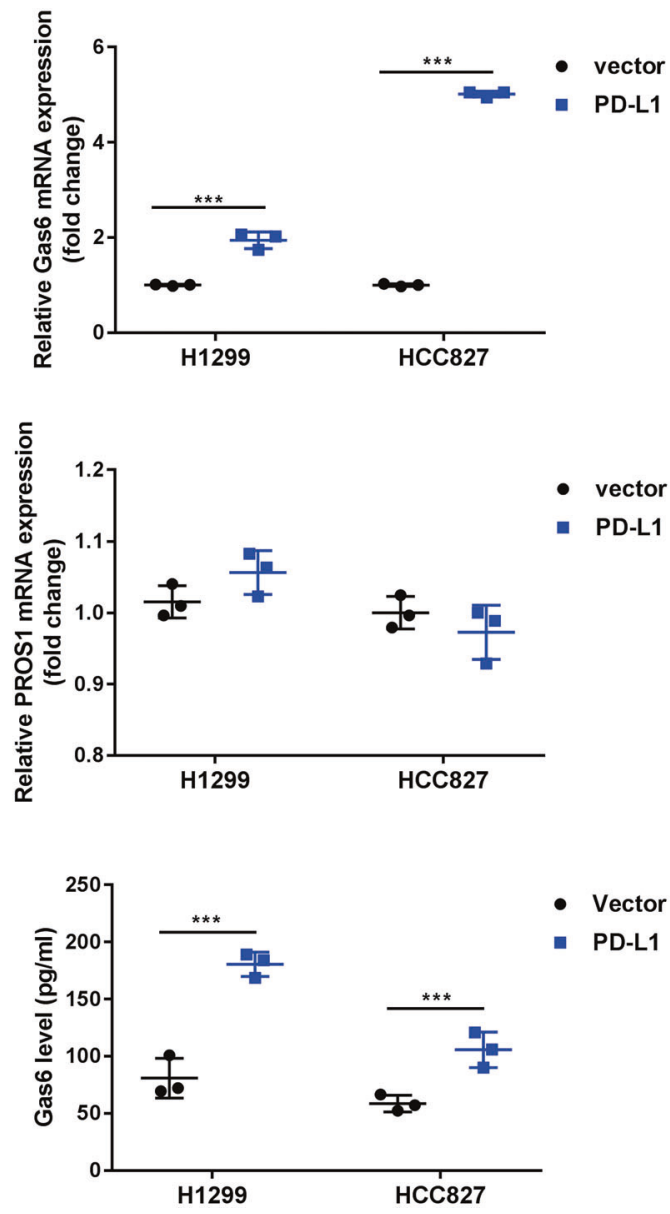

control cells. Data were presented as the mean \pm SD. Data were analysed using non-paired Student's $t$ test, one-way ANOVA analysis followed by Bonferroni's post hoc test. $* * P<0.01$; ***P<0.001 vs. control or as indicated.

\section{NPD-L1 mediates Gas6 transcription via interaction with the transcription factor Sp1}

Although nPD-L1 was reported to be discovered in the nucleus, no studies have been conducted to explore the function of nPD-L1 as a transcription factor. So next we speculated whether nPD-L1 can cooperate with various transcription factors to promote Gas6 transcription. First,

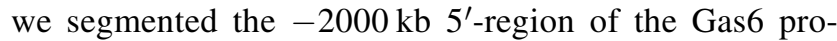
moter and constructed the corresponding luciferase reporter plasmids. Sequence range from -1504 to -967 $\mathrm{bp}$ in the Gas 6 promoter region showed increased luciferase activity in PD-L1-overexpressed cell line, suggesting this region is indispensable for the PD-L1-induced Gas6 promoter activity (Fig. 8a). Sequence analysis indicated that three potential $\mathrm{Sp} 1$ binding sites were located at the region from -1504 to -967 . Mutation of the third or all Sp1 binding sites abrogated the PD-L1induced luciferase activity, suggesting Sp1 is essential for 
A

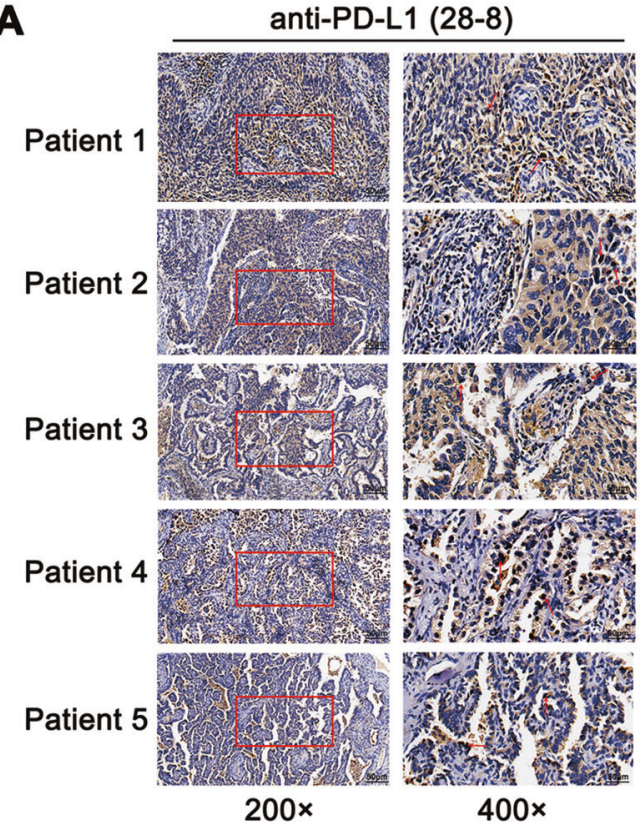

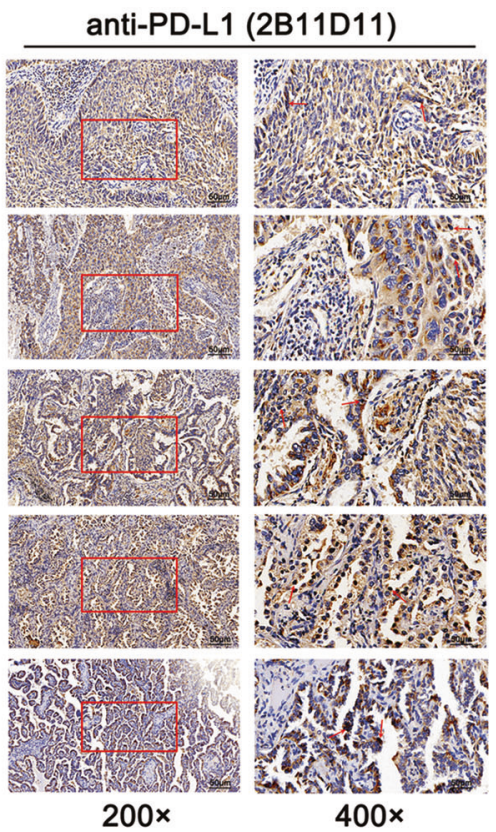

D

C
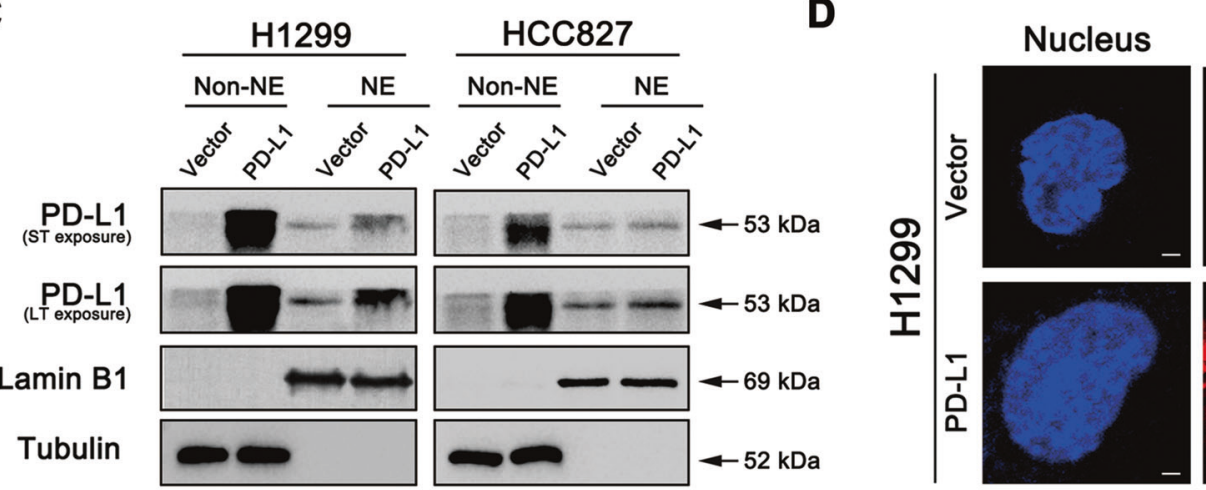

\section{PD-L1}

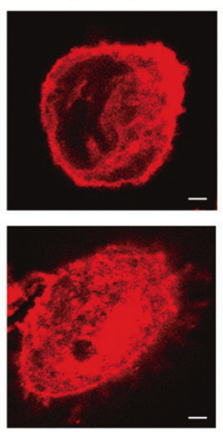

Merge
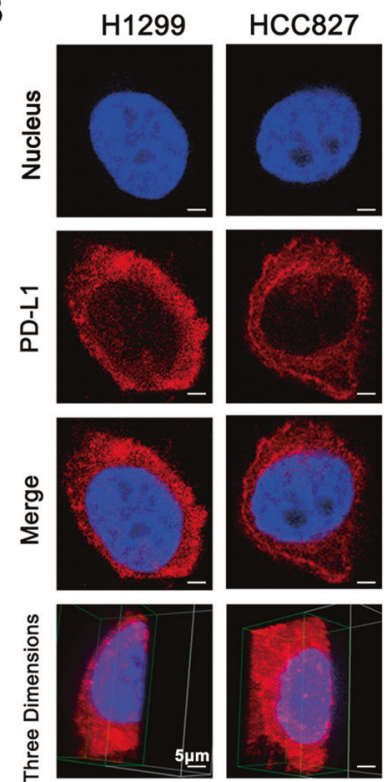

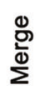
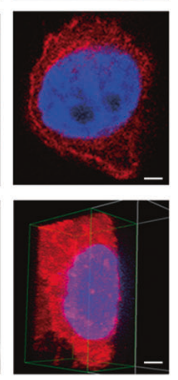

Nucleus
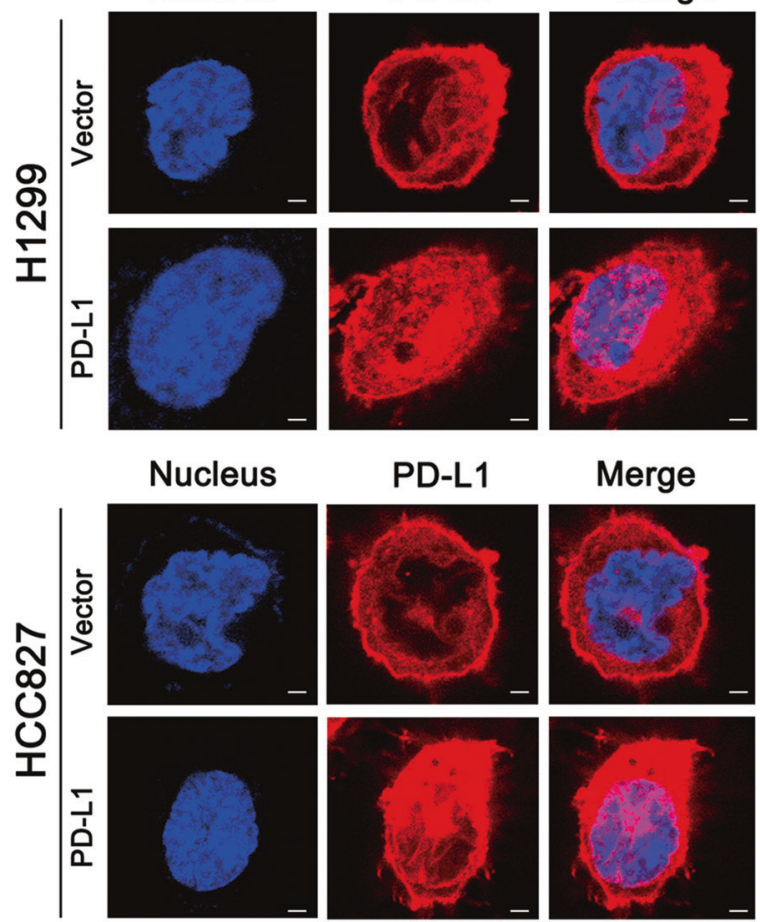

Merge
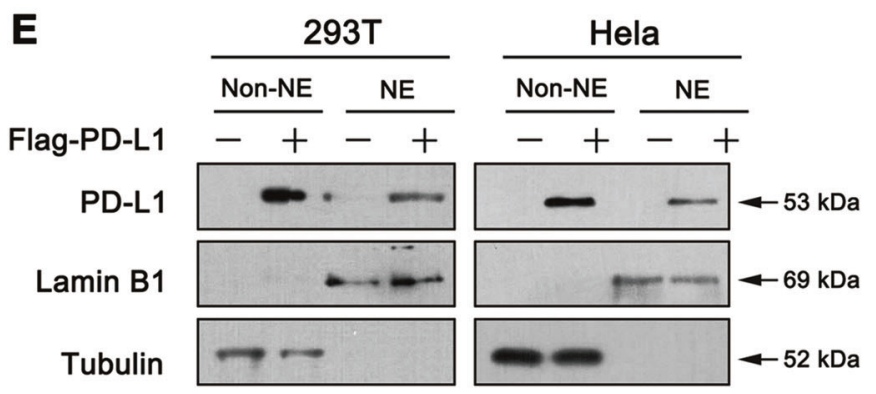

Fig. 6 PD-L1 overexpression can promote its nuclear translocation. a IHC assay of nPD-L1 staining with two separate anti-PD-L1 clones $(28-8$; 2B11D11) on the serial histological section of 24 paired NSCLC tissues. Five cases were selected as representative images. And the red arrows indicated positive nPD-L1 staining (Scale bar: $50 \mu \mathrm{m}$ ). b Immunofluorescence staining of nPD-L1 location in H1299 and HCC827 cell lines analysed by confocal microscopy (Scale bar: $5 \mu \mathrm{m})$. c PD-L1-overexpressed cells and control cells were subjected to

cellular fractionation followed by western blotting for PD-L1. Tubulin and Lamin B1 were the endogenous markers for cytosolic and nuclear proteins. d Immunofluorescence staining of nPD-L1 location in PDL1-overexpressed cells compared to control cells (Scale bar: $5 \mu \mathrm{m}$ ). e HEK293T and Hela cells were transfected with the Flag-PD-L1 or control plasmids and then subjected to western blot. NE nuclear extract. 
A

\begin{tabular}{ccc} 
Protein & PSMs & Peptides \\
\hline KPNB1 & 150 & 31 \\
KRT2 & 147 & 45 \\
BZW1 & 64 & 28 \\
ATXN10 & 55 & 21 \\
TNPO3 & 36 & 22 \\
IPO11 & 28 & 20
\end{tabular}

D

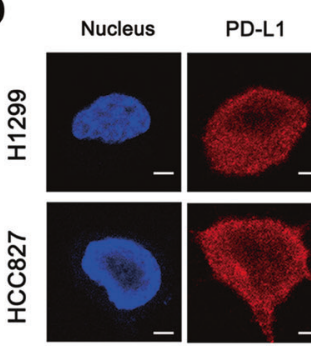

$\mathbf{F}$

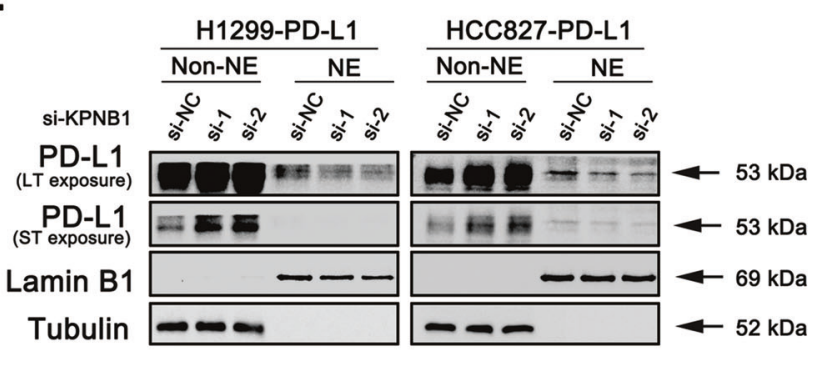

H

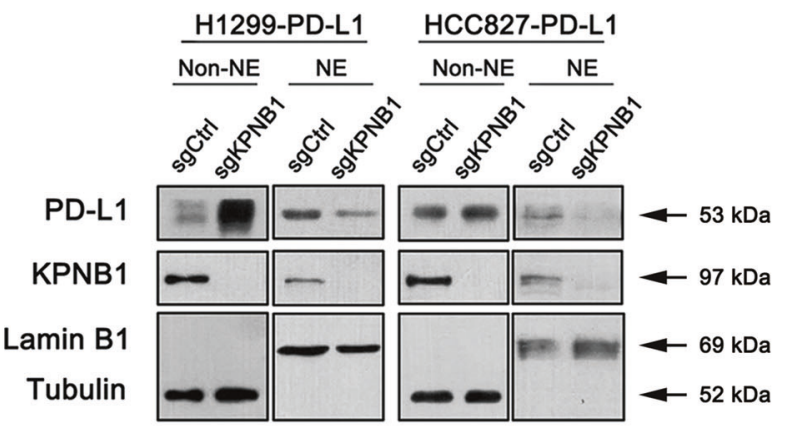

I

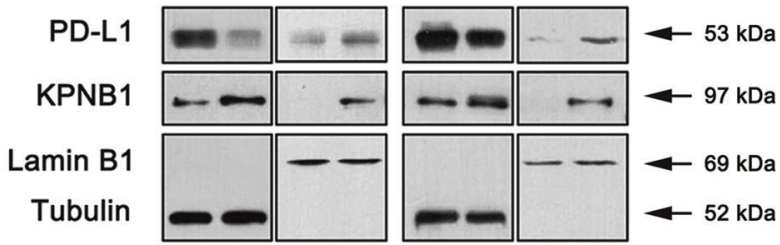

PD-L1-induced Gas6 promoter activity (Fig. 8b). Moreover, ChIP assay showed significant enrichment of both PD-L1 and Sp1 at the Gas6 promoter (Fig. 8c). We next tested whether $\mathrm{Sp} 1$ regulated Gas6 expression. Sp1

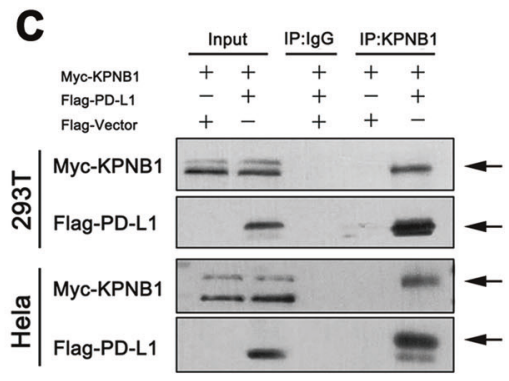

E
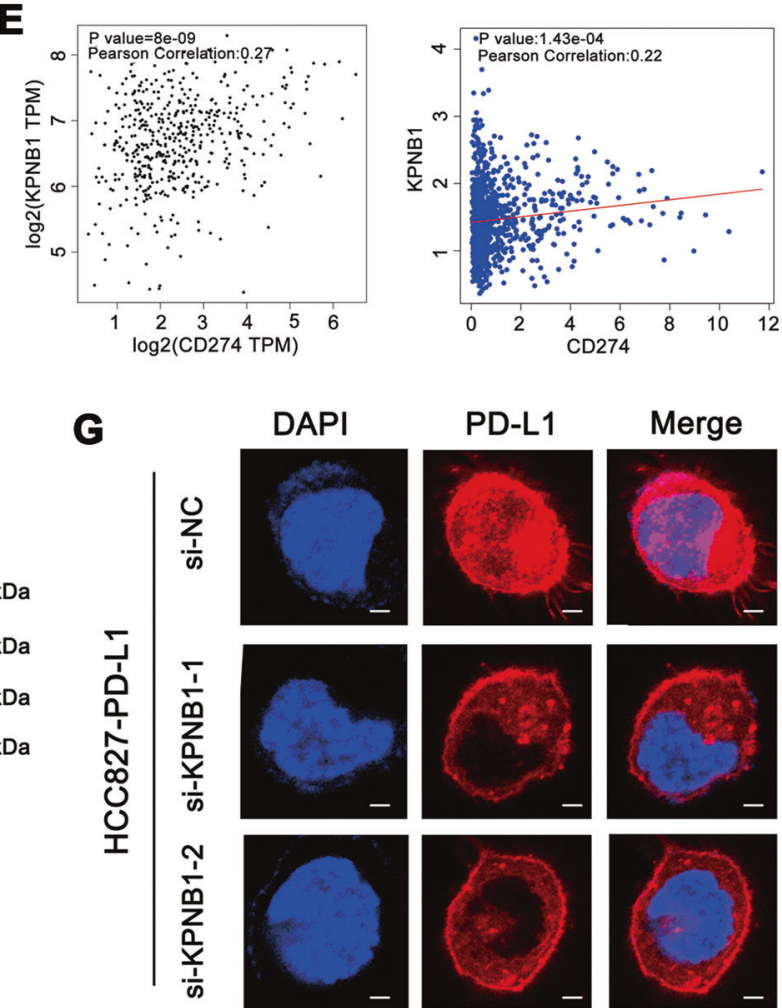

DAPI

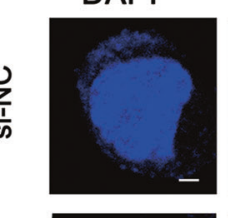

PD-L1
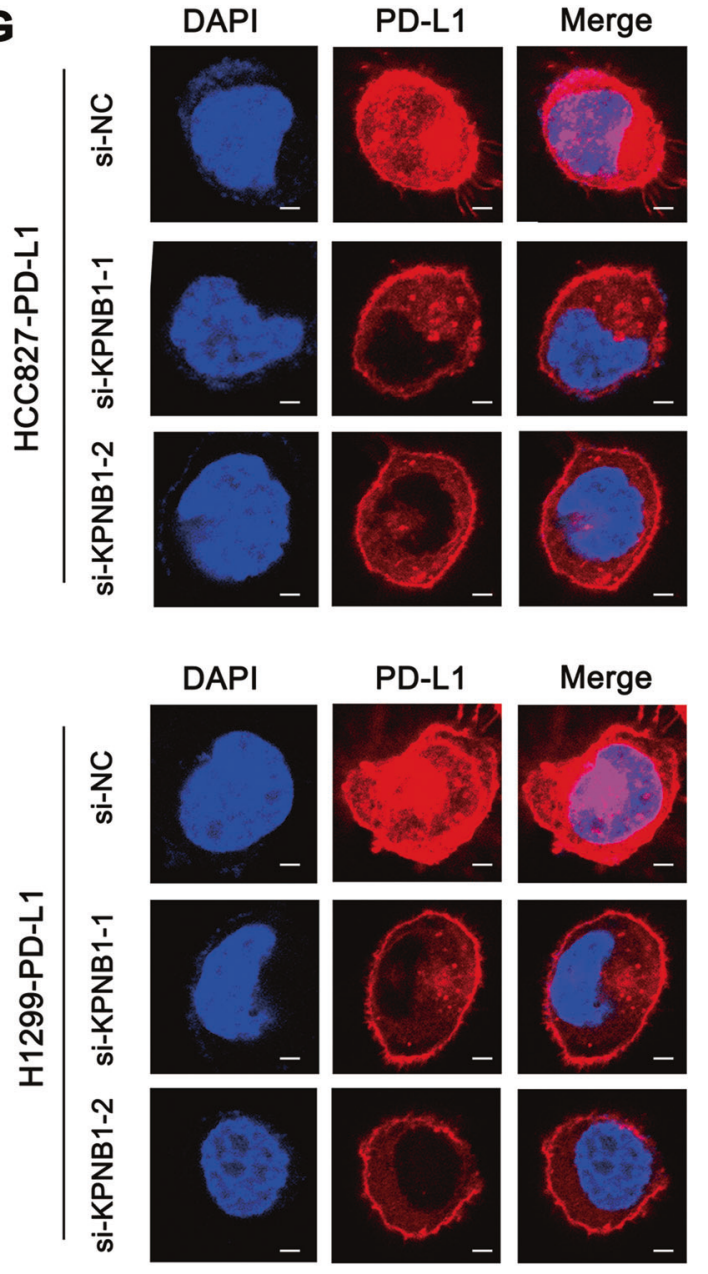

knockdown led to the reduced Gas6 expression, whereas overexpression of Sp1 increased Gas6 mRNA and protein expression levels (Fig. 8d, e). Furthermore, we demonstrated that the PD-L1-induced up-regulation of Gas6 
Fig. 7 KPNB1 is responsible for PD-L1 nuclear translocation. a The mass spectrometry assay of the potential PD-L1-interacting proteins. b Endogenous KPNB1 and PD-L1 interaction were detected in NSCLC cells using anti-KPNB1 and anti-PD-L1 antibodies for coIP and Western blot assays. c HEK293T and Hela cells were transfected with the indicated plasmids. Western blot and co-IP assays were performed to assess the interaction between PD-L1 and KPNB1. $\mathbf{d}$ The cellular location of PD-L1 and KPNB1 examined by confocal microscopy (Scale bar: $5 \mu \mathrm{m}$ ). e Correlation assay of PD-L1 and KPNB1 expression in NSCLC patient samples based on linkedomics and GEPIA database. $\mathbf{f}, \mathbf{h}$ Western blot assay of cytoplasmic and nuclear PD-L1 expression after KPNB1 knockdown with two specific siRNAs or knockout with CRISPR-Cas9 gene editing system. g Immunofluorescence staining of nPD-L1 location after KPNB1 knockdown in PD-L1-overexpressed cells (Scale bar: $5 \mu \mathrm{m}$ ). i Western blot assay of nPD-L1 expression in KPNB1-overexpressed cells.

mRNA expression was abrogated in the Sp1 knockdown condition but not with the control siRNA (Fig. 8g). Consistent with the above result, combination of PD-L1 and $\mathrm{Sp} 1$ synergistically enhanced the Gas6 promoter activity than applying PD-L1 or Sp1 alone (Fig. 8f). Together, all the above data showed that PD-L1 regulates the expression of Gas6 by cooperating with transcription factor Sp1.

\section{Gas6/MerTK signaling promotes NSCLC cell proliferation}

We have previously reported that PD-L1 can regulate Gas6 transcription and secretion, so next we mainly focussed on the role of Gas6/MerTK signaling it played in cell proliferation. Treatment of exogenous rh-Gas6 promoted the cell proliferation in $\mathrm{H} 1299$ and $\mathrm{HCC} 827$ cell lines, and the effect of cell proliferation was mediated by the engagement of MerTK signaling as knockdown of MerTK inhibited Gas6-induced proliferation (Fig. 9a). Western blot results revealed increased protein levels of p-MerTK, p-AKT and p-Erk after Gas6 stimulation, while this up-regulation was inhibited after silencing MerTK expression (Fig. 9b).

\section{Discussion}

The role of PD-L1 in mediating tumour cells escape from immune surveillance has been widely discussed. Accumulating clinical trials have demonstrated the promising outcome in prolonging the long-term and durable overall survival in advanced NSCLC patients [36-38]. However, only $20-30 \%$ of NSCLC patients responded to the immunotherapy targeting PD-1/PD-L1 checkpoints [39]. And some patients even with higher PD-L1 expression still cannot benefit from the immunotherapy. Recent studies have also highlighted the role of tumour intrinsic PD-L1 in promoting cell proliferation, metastasis, drug resistance, metabolism and other effects independent of the immune system [8-13]. Here we first demonstrated that partial PDL1 translocated into the nucleus to further regulate Gas6

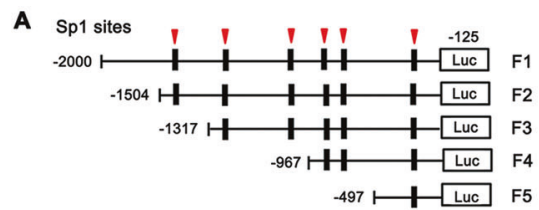

C

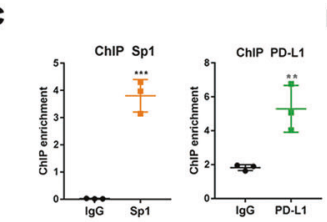

D
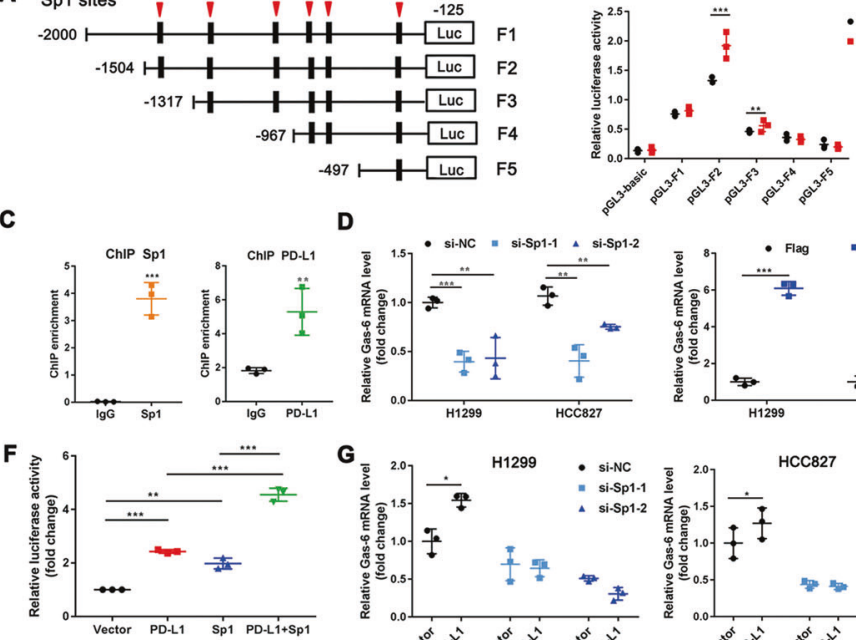

- Vector
PD.L1
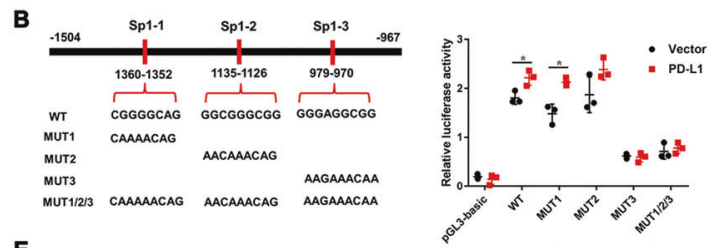

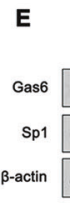
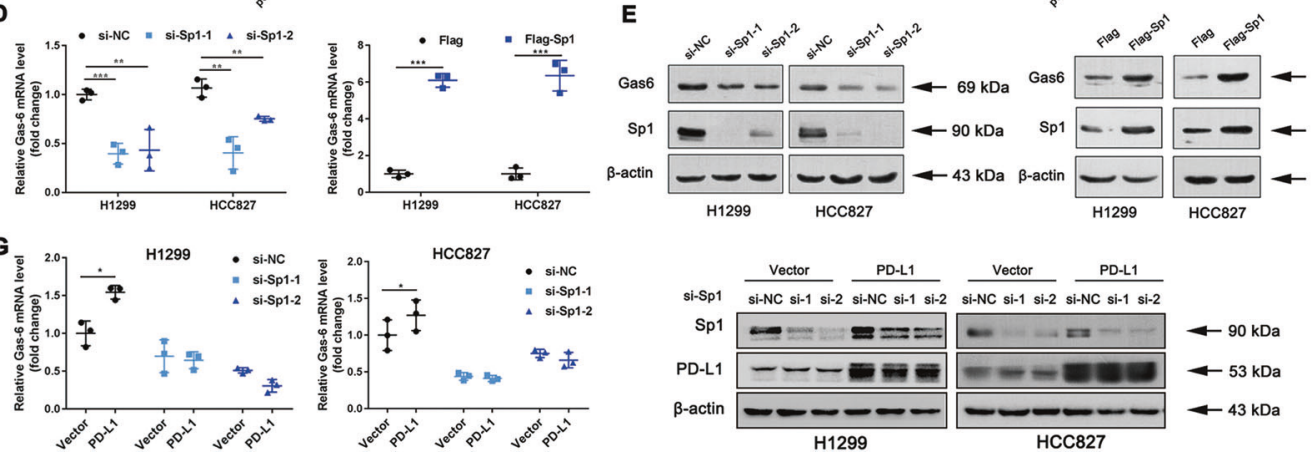
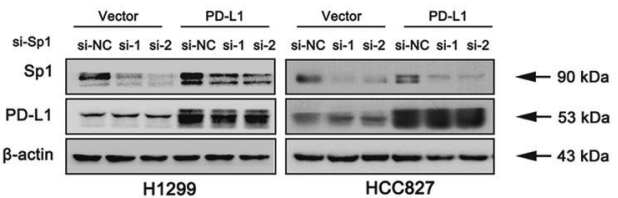

Fig. 8 PD-L1 cooperates with SP1 to regulate Gas6 transcription. a, b The results of the luciferase reporter assay in H1299-PD-L1overexpressed cells and control cells after transfection with a set of luciferase reporter plasmids containing different regions of the Gas6 promoter or mutants at three SP1 binding sites for $24 \mathrm{~h}$. c ChIP assays were performed to detect PD-L1 and Sp1 binding to the Gas6 promoter in H1299-PD-L1-overexpressed cells and control cells. d, e The mRNA and protein levels of Gas6 in H1299 and HCC827 cells after transient transfection with Sp1 siRNAs or Sp1-overexpressing plasmids. f The luciferase activity of the Gas6 promoter in 293 cells transfected with control vector, PD-L1 vector, Sp1 vector or both PD-L1 and Sp1 vectors. g H1299- and HCC827-PD-L1overexpressed cells were silenced with si-Sp1 or si-NC (control). Data were presented as the mean $\pm \mathrm{SD}$. Data were analysed using nonpaired Student's $t$ test, one-way or two-way ANOVA analysis followed by Bonferroni's post hoc test. $* P<0.05$; $* * P<0.01$; ***P< 0.001 vs. control or as indicated. 


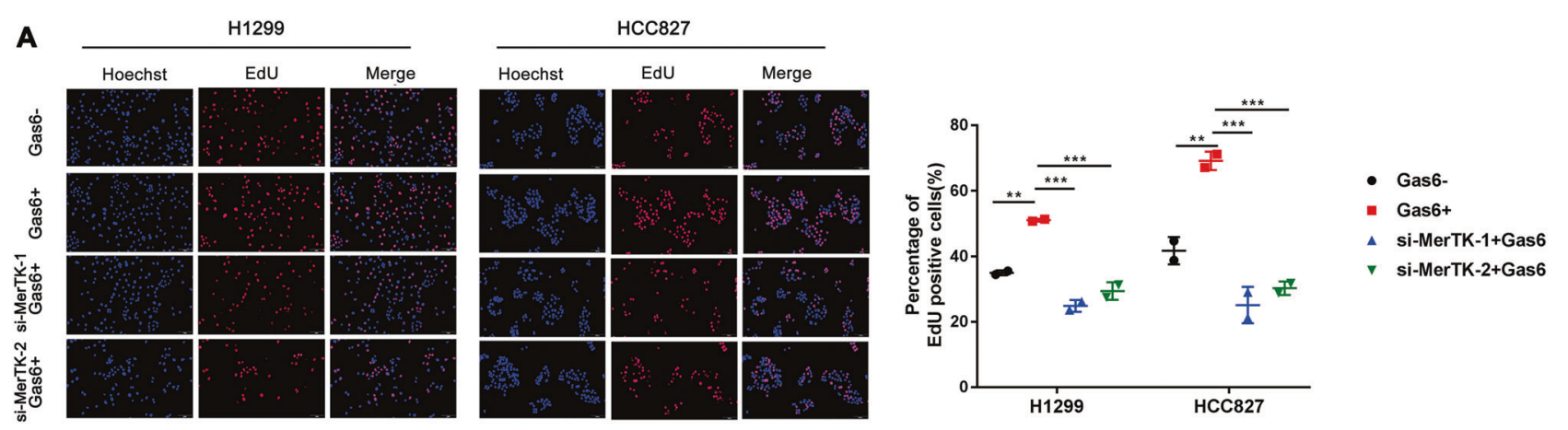

B

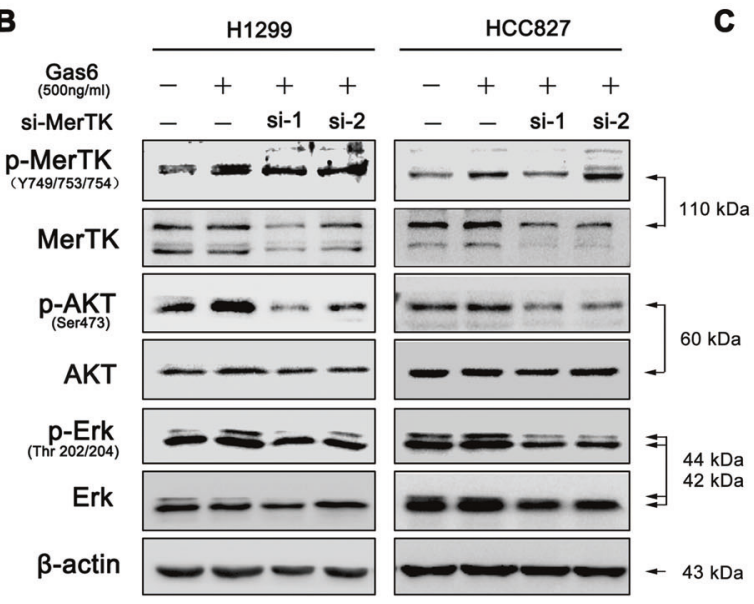

Fig. 9 Gas6/MerTK signaling promotes NSCLC cell proliferation. a EdU assay was performed in $\mathrm{H} 1299$ and $\mathrm{HCC} 827$ cells transfected with si-MerTK or si-NC with or without Gas6 stimulation for $24 \mathrm{~h}$ (Scale bar: $2 \mathrm{~mm}$ ). b Western blot assay of p-MerTK, MerTK, p-AKT, AKT, p-Erk and Erk. c The proposed mechanistic model underlying

expression and thus activate MerTK signaling pathway to promote NSCLC cell proliferation.

NPD-L1 has been implicated in mediating the drug resistance of breast cancer cell and functions as a prognostic indicator in prostate cancer and colorectal cancer patients $[16,17]$. It is also reported that nPD-L1 competes with WAPL for binding to PDS5B, and regulates breast cancer cell sister chromatid cohesion [40]. Here, our study confirmed the positive nPD-L1 expression in NSCLC tissues and cell lines. Recently, the underlying mechanism behind PD-L1 nuclear translocation has been explored in breast cancer. Under hypoxia treatment, p-Stat3 physically interacts with PD-L1 and facilitates its nuclear translocation [41]. Another report found that deacetylation of PDL1 at Lys 263 by HDAC2 triggers nuclear translocation by interacting with multiple proteins that are involved in endocytosis and nuclear import like HIP1R, vimentin [42]. Here in our study, we found that KPNB1 was the most relevant protein with PD-L1. Moreover, KPNB1 participates in regulating the cell cycle, which is essential for cancer cell proliferation [43-45]. The Oncomine database (https://www.oncomine.org/) indicated higher KPNB1

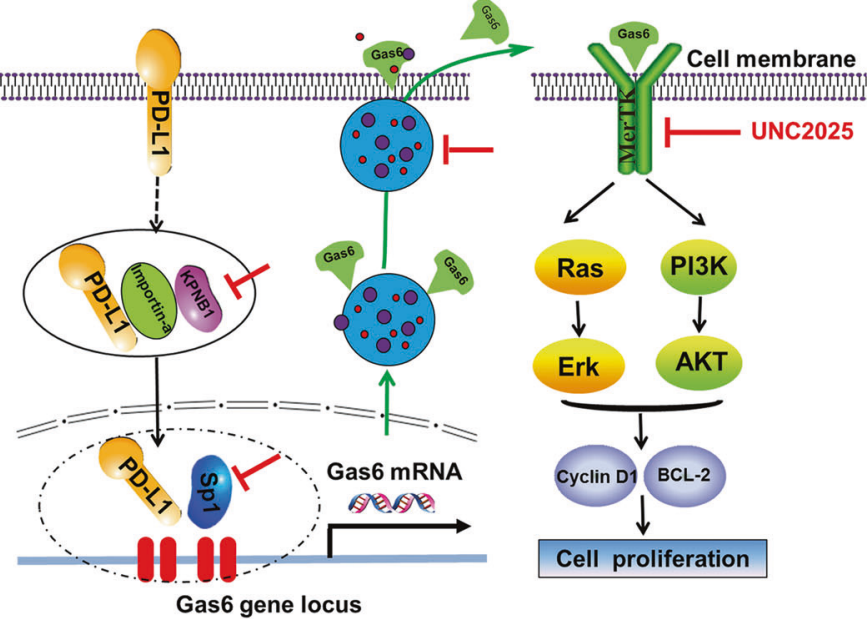

the nPD-L1-mediated Gas6/MerTK signaling stimulating NSCLC cell proliferation. Data were presented as the mean \pm SD. Data were analysed using one-way ANOVA analysis followed by Bonferroni's post hoc test. $* * P<0.01 ; * * * P<0.001$ vs. control or as indicated.

expression in lung cancer tissues, implying poorer overall survival and progression-free survival (http://www. kmplot.com/) (Fig. S7). We found that knockdown of KPNB1 blocked PD-L1 nuclear import, implying that KPNB1 was critical for PD-L1 nuclear translocation. However, further studies are needed to better clarify the functional-binding domains between PD-L1 and KPNB1. Besides, we need to determine the source of nPD-L1 whether from cell membrane or from cytoplasm.

No studies have shown that PD-L1 contains a DNAbinding domain and thus functions as a transcription factor. Recent new findings showed that nPD-L1 and p-Y705-Stat3 acted as co-activators in regulating GSDMC mRNA expression and then switched apoptosis to pyroptosis [41]. Moreover, nPD-L1 interacted with STAT3, c-Jun to govern immune-response-related genes expression and thereby modulated the anti-tumour immune response [42]. Sp1 is well identified as a common transcription factor that recognises the GC-rich DNA sequences in the promoter regions of various genes [46, 47]. Here our data demonstrated strong $\mathrm{Sp} 1$ enrichment at the promoter region of Gas6. And Sp1, together with nPD-L1, acted as 
co-activators to regulate Gas6 transcription. While there is a possibility that other transcription factors may participate in Gas6 transcription since $\mathrm{Sp} 1$ is reported to have overlapping function with other $\mathrm{Sp} / \mathrm{KLF}$ family members, such as Sp3 [48]. To our surprise, a connection between PD-L1 and Sp1 was found as PD-L1 can regulate Sp1 at mRNA and protein levels (Fig. S8). It is reported that the interaction between proteins and $\mathrm{Sp} 1$ depends on the $\mathrm{Sp} 1$ zinc finger DNAbinding domain (ZFDBD) [49]. Therefore, whether PD-L1 directly interacts with the ZFDBD of Sp1 still remains unclarified. Surprisingly, we further found that the mRNA levels of Sp1-regulated targets $C D K 1, C D K 4$ and HOGG1 were up-regulated after PD-L1 overexpression, suggesting that PD-L1 might regulate other Sp1 targets as well $[50,51]$. Therefore, we speculate that PD-L1 could interact with $\mathrm{Sp} 1$ to form protein complex to function as transcriptional regulators.

MerTK was first recognised for its role in mediating the phagocytosis of apoptotic cells, which negatively regulating the immune system [52]. Recent studies also indicated high MerTK expression in various tumour tissues, and the activation of MerTK signaling contributed to tumour progression [53]. Studies showed that PS-induced MerTK activation led to PD-L1 up-regulation in breast cancer cells [54]. Another study suggested that MerTK overexpression in epithelial cancer cells drives efferocytosis via increasing PD-L1 expression [55]. Although these studies showed that MerTK induced PD-L1 up-regulation, interestingly, our data found that MerTK activation was regulated by PD-L1 in both NSCLC cell lines and patient samples. Moreover, we identified that PD-L1 can regulate cell cycle and apoptosis to promote cell proliferation via the MerTK signaling pathway. In addition, it would be interesting to clarify whether nPD-L1-mediated Gas6/MerTK signaling activation may account for the compromised immunotherapy response which targeting PD-L1/PD-1 checkpoints in patients with higher PD-L1 levels, which needs to be further investigated.

In summary, our study for the first time demonstrated that PD-L1 can regulate NSCLC proliferation via Gas6/ MerTK signaling. Furthermore, we identified KPNB1 as the cytoplasm-to-nucleus shuttle for PD-L1 and established a novel role for nPD-L1 in promoting Gas6 transcription via the transcription factor Sp1 (Fig. 9c). These data redefine the function of nPD-L1 in tumour, and we speculate that targeting KPNB1 or MerTK signaling pathway in combination with PD-L1 immunotherapy may improve the clinical outcome of NSCLC patients.

Acknowledgements We thank all patients who participated in this study for their cooperation. We also thank the native English-speaking scientists of the Springer Nature Company for editing our paper. This work was supported by grants from the Jiangsu Provincial Medical Youth Talent (No. QNRC2016746 to Z-YL), the National Natural
Science Foundation of China (81802885, 81702870), the Suzhou Key Laboratory for Respiratory Medicine (No. SZS201617), the Clinical Medical Center of Suzhou (No. Szzx201502), the Jiangsu Provincial Key Medical Discipline (No. ZDXKB2016007).

\section{Compliance with ethical standards}

Conflict of interest The authors declare that they have no conflict of interest.

Publisher's note Springer Nature remains neutral with regard to jurisdictional claims in published maps and institutional affiliations.

Open Access This article is licensed under a Creative Commons Attribution 4.0 International License, which permits use, sharing, adaptation, distribution and reproduction in any medium or format, as long as you give appropriate credit to the original author(s) and the source, provide a link to the Creative Commons license, and indicate if changes were made. The images or other third party material in this article are included in the article's Creative Commons license, unless indicated otherwise in a credit line to the material. If material is not included in the article's Creative Commons license and your intended use is not permitted by statutory regulation or exceeds the permitted use, you will need to obtain permission directly from the copyright holder. To view a copy of this license, visit http://creativecommons. org/licenses/by/4.0/.

\section{References}

1. Sanmamed MF, Chen L. A paradigm shift in cancer immunotherapy: from enhancement to normalization. Cell. 2019; 176:677.

2. Sun C, Mezzadra R, Schumacher TN. Regulation and Function of the PD-L1 Checkpoint. Immunity. 2018;48:434-52.

3. Gandara DR, Paul SM, Kowanetz M, Schleifman E, Zou W, Li Y, et al. Blood-based tumor mutational burden as a predictor of clinical benefit in non-small-cell lung cancer patients treated with atezolizumab. Nat Med. 2018;24:1441-8.

4. Gettinger S, Horn L, Jackman D, Spigel D, Antonia S, Hellmann $\mathrm{M}$, et al. Five-Year Follow-Up of Nivolumab in Previously Treated Advanced Non-Small-Cell Lung Cancer: results From the CA209-003 Study. J Clin Oncol. 2018;36:1675-84.

5. Dong P, Xiong Y, Yue J, Hanley SJB, Watari H. Tumor-Intrinsic PD-L1 Signaling in Cancer Initiation, Development and Treatment: beyond immune evasion. Front Oncol. 2018;8:386.

6. Escors D, Gato-Canas M, Zuazo M, Arasanz H, Garcia-Granda MJ, Vera R, et al. The intracellular signalosome of PD-L1 in cancer cells. Signal Transduct Target Ther. 2018;3:26.

7. Azuma T, Yao S, Zhu G, Flies AS, Flies SJ, Chen L. B7-H1 is a ubiquitous antiapoptotic receptor on cancer cells. Blood 2008; 111:3635-43.

8. Qiu XY, Hu DX, Chen WQ, Chen RQ, Qian SR, Li CY, et al. PDL1 confers glioblastoma multiforme malignancy via Ras binding and Ras/Erk/EMT activation. Biochim Biophys Acta Mol Basis Dis. 2018;1864:1754-69.

9. Zhang Y, Zeng Y, Liu T, Du W, Zhu J, Liu Z, et al. The canonical TGF-beta/Smad signalling pathway is involved in PD-L1-induced primary resistance to EGFR-TKIs in EGFR-mutant non-small-cell lung cancer. Respir Res. 2019;20:164.

10. Takada K, Toyokawa G, Okamoto T, Baba S, Kozuma Y, Matsubara $\mathrm{T}$, et al. Metabolic characteristics of programmed cell death-ligand 1-expressing lung cancer on (18) Ffluorodeoxyglucose positron emission tomography/computed tomography. Cancer Med. 2017;6:2552-61. 
11. Feng D, Qin B, Pal K, Sun L, Dutta S, Dong H, et al. BRAF (V600E)-induced, tumor intrinsic PD-L1 can regulate chemotherapy-induced apoptosis in human colon cancer cells and in tumor xenografts. Oncogene 2019;38:6752-66.

12. Gupta HB, Clark CA, Yuan B, Sareddy G, Pandeswara S, Padron AS,et al. Tumor cell-intrinsic PD-L1 promotes tumor-initiating cell generation and functions in melanoma and ovarian cancer. Signal Transduct Target Ther. 2016;1:16030.

13. Zhang N, Zeng Y, Du W, Zhu J, Shen D, Liu Z, et al. The EGFR pathway is involved in the regulation of PD-L1 expression via the IL-6/JAK/STAT3 signaling pathway in EGFR-mutated non-small cell lung cancer. Int J Oncol. 2016;49:1360-8.

14. Parra ER, Villalobos P, Rodriguez-Canales J. The Multiple Faces of Programmed Cell Death Ligand 1 Expression in Malignant and Nonmalignant Cells. Appl Immunohistochem Mol Morphol. 2019;27:287-94.

15. Chen L, Deng H, Lu M, Xu B, Wang Q, Jiang J, et al. B7-H1 expression associates with tumor invasion and predicts patient's survival in human esophageal cancer. Int J Clin Exp Pathol. 2014;7:6015-23.

16. Satelli A, Batth IS, Brownlee Z, Rojas C, Meng QH, Kopetz S, et al. Potential role of nuclear PD-L1 expression in cell-surface vimentin positive circulating tumor cells as a prognostic marker in cancer patients. Sci Rep. 2016;6:28910.

17. Ghebeh H, Lehe C, Barhoush E, Al-Romaih K, Tulbah A, AlAlwan M, et al. Doxorubicin downregulates cell surface B7-H1 expression and upregulates its nuclear expression in breast cancer cells: role of B7-H1 as an anti-apoptotic molecule. Breast Cancer Res. 2010;12:R48.

18. Schulz D, Streller M, Piendl G, Brockhoff G, Reichert TE, Menevse AN, et al. Differential localization of PD-L1 and Akt-1 involvement in radioresistant and radiosensitive cell lines of head and neck squamous cell carcinoma. Carcinogenesis. 2019;41: 984-92.

19. Palma M, Riffo EN, Suganuma T, Washburn MP, Workman JL, Pincheira R, et al. Identification of a nuclear localization signal and importin beta members mediating NUAK1 nuclear import inhibited by oxidative stress. J Cell Biochem. 2019;120: 16088-107.

20. Sheng C, Qiu J, He Z, Wang H, Wang Q, Guo Z, et al. Suppression of Kpnbeta1 expression inhibits human breast cancer cell proliferation by abrogating nuclear transport of Her2. Oncol Rep. 2018;39:554-64.

21. Wang T, Huang Z, Huang N, Peng Y, Gao M, Wang X, et al. Inhibition of KPNB1 Inhibits Proliferation and Promotes Apoptosis of Chronic Myeloid Leukemia Cells Through Regulation of E2F1. Onco Targets Ther. 2019;12:10455-67.

22. Zhang Y, Li KF. Karyopherin betal deletion suppresses tumor growth and metastasis in colorectal cancer (CRC) by reducing MET expression. Biomed Pharmacother. 2019;120:109127.

23. Wang H, Wang D, Li C, Zhang X, Zhou X, Huang J. High Kpnbeta1 expression promotes non-small cell lung cancer proliferation and chemoresistance via the PI3-kinase/AKT pathway. Tissue Cell. 2018;51:39-48.

24. Burstyn-Cohen T. TAM receptor signaling in development. Int $\mathbf{J}$ Dev Biol. 2017;61:215-24.

25. Wu G, Ma Z, Cheng Y, Hu W, Deng C, Jiang S, et al. Targeting Gas6/TAM in cancer cells and tumor microenvironment. Mol Cancer. 2018;17:20.

26. van der Meer JH, van der Poll T, van 't Veer C. TAM receptors, Gas6, and protein S: roles in inflammation and hemostasis. Blood 2014;123:2460-9.

27. Myers KV, Amend SR, Pienta KJ. Targeting Tyro3, Axl and MerTK (TAM receptors): implications for macrophages in the tumor microenvironment. Mol Cancer 2019;18:94.
28. Eom H, Kaushik N, Yoo KC, Shim JK, Kwon M, Choi MY, et al. MerTK mediates STAT3-KRAS/SRC-signaling axis for glioma stem cell maintenance. Artif Cells Nanomed Biotechnol. 2018; 46:87-95.

29. Wu J, Frady LN, Bash RE, Cohen SM, Schorzman AN, Su YT, et al. MerTK as a therapeutic target in glioblastoma. Neuro Oncol. 2018;20:92-102.

30. Shi C, Li X, Wang X, Ding N, Ping L, Shi Y, et al. The protooncogene Mer tyrosine kinase is a novel therapeutic target in mantle cell lymphoma. J Hematol Oncol. 2018;11:43.

31. Sinik L, Minson KA, Tentler JJ, Carrico J, Bagby SM, Robinson WA, et al. Inhibition of MERTK Promotes Suppression of Tumor Growth in BRAF Mutant and BRAF Wild-Type Melanoma. Mol Cancer Ther. 2019;18:278-88.

32. Yan D, Parker RE, Wang X, Frye SV, Earp HS 3rd, DeRyckere D, et al. MERTK Promotes Resistance to Irreversible EGFR Tyrosine Kinase Inhibitors in Non-small Cell Lung Cancers Expressing Wildtype EGFR Family Members. Clin Cancer Res. 2018;24:6523-35.

33. Zhu J, Zeng Y, Li W, Qin H, Lei Z, Shen D, et al. CD73/NT5E is a target of miR-30a-5p and plays an important role in the pathogenesis of non-small cell lung cancer. Mol Cancer. 2017;16:34.

34. Tang H, Zhu J, Du W, Liu S, Zeng Y, Ding Z, et al. CPNE1 is a target of miR-335-5p and plays an important role in the pathogenesis of non-small cell lung cancer. J Exp Clin Cancer Res. 2018;37:131.

35. Geng K, Kumar S, Kimani SG, Kholodovych V, Kasikara C, Mizuno K, et al. Requirement of Gamma-Carboxyglutamic Acid Modification and Phosphatidylserine Binding for the Activation of Tyro3, Axl, and Mertk Receptors by Growth Arrest-Specific 6. Front Immunol. 2017;8:1521.

36. Antonia SJ, Borghaei H, Ramalingam SS, Horn L, De Castro Carpeno J, Pluzanski A, et al. Four-year survival with nivolumab in patients with previously treated advanced non-small-cell lung cancer: a pooled analysis. Lancet Oncol. 2019;20:1395-408.

37. Mok TSK, Wu YL, Kudaba I, Kowalski DM, Cho BC, Turna HZ, et al. Pembrolizumab versus chemotherapy for previously untreated, PD-L1-expressing, locally advanced or metastatic nonsmall-cell lung cancer (KEYNOTE-042): a randomised, openlabel, controlled, phase 3 trial. Lancet 2019;393:1819-30.

38. Sacher AG, Gandhi L. Biomarkers for the Clinical Use of PD-1/ PD-L1 Inhibitors in Non-Small-Cell Lung Cancer: a Review. JAMA Oncol. 2016;2:1217-22.

39. Feng H, Zhao Y, Li M, Lin J, Zhang J, Ma J, et al. Efficacy and safety of anti PD-1/PD-L1 in the treatment of advanced nonsmall-cell lung cancer: a single arm meta-analysis. Chinese. J Evid Based Med. 2018;18:322-33.

40. Yu J, Qin B, Moyer AM, Nowsheen S, Tu X, Dong H, et al. Regulation of sister chromatid cohesion by nuclear PD-L1. Cell Res. 2020;30:590-601.

41. Hou J, Zhao R, Xia W, Chang CW, You Y, Hsu JM, et al. PD-L1mediated gasdermin $\mathrm{C}$ expression switches apoptosis to pyroptosis in cancer cells and facilitates tumour necrosis. Nat Cell Biol. 2020;22:1264-75.

42. Gao Y, Nihira NT, Bu X, Chu C, Zhang J, Kolodziejczyk A, et al. Acetylation-dependent regulation of PD-L1 nuclear translocation dictates the efficacy of anti-PD-1 immunotherapy. Nat Cell Biol. 2020;22:1064-75.

43. Di Girolamo M. Regulation of nucleocytoplasmic transport by ADP-ribosylation: the emerging role of karyopherin-beta1 monoADP-ribosylation by ARTD15. Curr Top Microbiol Immunol. 2015;384:189-209.

44. Lu T, Bao Z, Wang Y, Yang L, Lu B, Yan K, et al. Karyopherinbeta1 regulates proliferation of human glioma cells via Wnt/ beta-catenin pathway. Biochem Biophys Res Commun. 2016; 478:1189-97. 
45. Zhu ZC, Liu JW, Yang C, Li MJ, Wu RJ, Xiong ZQ. Targeting KPNB1 overcomes TRAIL resistance by regulating DR5, Mcl-1 and FLIP in glioblastoma cells. Cell Death Dis. 2019;10:118.

46. O'Connor L, Gilmour J, Bonifer C. The Role of the Ubiquitously Expressed Transcription Factor Sp1 in Tissue-specific Transcriptional Regulation and in Disease. Yale J Biol Med. 2016;89: $513-25$.

47. Meinders M, Kulu DI, van de Werken HJ, Hoogenboezem M, Janssen $\mathrm{H}$, Brouwer RW, et al. Sp1/Sp3 transcription factors regulate hallmarks of megakaryocyte maturation and platelet formation and function. Blood 2015;125:1957-67.

48. Kaczynski J, Cook T, Urrutia R. Sp1- and Kruppel-like transcription factors. Genome Biol. 2003;4:206.

49. Lee DK, Suh D, Edenberg HJ, Hur MW. POZ domain transcription factor, FBI-1, represses transcription of ADH5/FDH by interacting with the zinc finger and interfering with DNA binding activity of Sp1. J Biol Chem. 2002;277:26761-8.

50. Deng YR, Chen XJ, Chen W, Wu LF, Jiang HP, Lin D, et al. Sp1 contributes to radioresistance of cervical cancer through targeting
G2/M cell cycle checkpoint CDK1. Cancer Manag Res. 2019; 11:5835-44.

51. Willoughby JA Sr., Sundar SN, Cheung M, Tin AS, Modiano J, Firestone GL. Artemisinin blocks prostate cancer growth and cell cycle progression by disrupting Sp1 interactions with the cyclindependent kinase-4 (CDK4) promoter and inhibiting CDK4 gene expression. J Biol Chem. 2009;284:2203-13.

52. Caberoy NB, Alvarado G, Bigcas JL, Li W. Galectin-3 is a new MerTK-specific eat-me signal. J Cell Physiol. 2012;227:401-407.

53. Vouri M, Hafizi S. TAM Receptor Tyrosine Kinases in Cancer Drug Resistance. Cancer Res. 2017;77:2775-8.

54. Kasikara C, Kumar S, Kimani S, Tsou WI, Geng K, Davra V, et al. Phosphatidylserine Sensing by TAM Receptors Regulates AKT-Dependent Chemoresistance and PD-L1 Expression. Mol Cancer Res. 2017;15:753-64.

55. Nguyen KQ, Tsou WI, Calarese DA, Kimani SG, Singh S, Hsieh $\mathrm{S}$, et al. Overexpression of MERTK receptor tyrosine kinase in epithelial cancer cells drives efferocytosis in a gain-of-function capacity. J Biol Chem. 2014;289:25737-49. 